\title{
Development of prognostic models for Health-Related Quality of Life following traumatic brain injury
}

\author{
Isabel R. A. Retel Helmrich ${ }^{1,8}$ - David van Klaveren ${ }^{1,2,8} \cdot$ Simone A. Dijkland $^{1,8} \cdot$ Hester F. Lingsma ${ }^{1,8}$. \\ Suzanne Polinder ${ }^{1,8} \cdot$ Lindsay Wilson $^{5} \cdot$ Nicole von Steinbuechel ${ }^{3} \cdot$ Joukje van der Naalt ${ }^{4} \cdot$ Andrew I. R. Maas $^{6}$. \\ Ewout W. Steyerberg ${ }^{1,7}$. CENTER-TBI Collaborators
}

Accepted: 25 June 2021 / Published online: 30 July 2021

(C) The Author(s) 2021

\begin{abstract}
Background Traumatic brain injury (TBI) is a leading cause of impairments affecting Health-Related Quality of Life (HRQoL). We aimed to identify predictors of and develop prognostic models for HRQoL following TBI.

Methods We used data from the Collaborative European NeuroTrauma Effectiveness Research in Traumatic Brain Injury (CENTER-TBI) Core study, including patients with a clinical diagnosis of TBI and an indication for computed tomography presenting within $24 \mathrm{~h}$ of injury. The primary outcome measures were the SF-36v2 physical (PCS) and mental (MCS) health component summary scores and the Quality of Life after Traumatic Brain Injury (QOLIBRI) total score 6 months post injury. We considered 16 patient and injury characteristics in linear regression analyses. Model performance was expressed as proportion of variance explained $\left(R^{2}\right)$ and corrected for optimism with bootstrap procedures.

Results 2666 Adult patients completed the HRQoL questionnaires. Most were mild TBI patients (74\%). The strongest predictors for PCS were Glasgow Coma Scale, major extracranial injury, and pre-injury health status, while MCS and QOLIBRI were mainly related to pre-injury mental health problems, level of education, and type of employment. $R^{2}$ of the full models was $19 \%$ for PCS, $9 \%$ for MCS, and 13\% for the QOLIBRI. In a subset of patients following predominantly mild TBI $(N=$ 436 ), including 2 week HRQoL assessment improved model performance substantially ( $R^{2}$ PCS $15 \%$ to $37 \%$, MCS $12 \%$ to $36 \%$, and QOLIBRI $10 \%$ to $48 \%$ ).

Conclusion Medical and injury-related characteristics are of greatest importance for the prediction of PCS, whereas patientrelated characteristics are more important for the prediction of MCS and the QOLIBRI following TBI.
\end{abstract}

Keywords Prognostic model research $\cdot$ Traumatic brain injury $\cdot$ Health-related quality of life $\cdot$ SF-36 $\cdot$ QOLIBRI

\begin{tabular}{|c|c|}
\hline \\
\hline \multirow{3}{*}{ CENTER-TBI } & Collaborative European NeuroTrauma \\
\hline & Effectiveness Research in Traumatic \\
\hline & Brain Injury \\
\hline GCS & Glasgow Coma Scale \\
\hline HRQoL & Health-Related Quality of Life \\
\hline MEI & Major extracranial injury \\
\hline MCS & Mental component summary score \\
\hline PCS & Physical component summary score \\
\hline PTSD & Post-traumatic stress disorder \\
\hline
\end{tabular}

Members of CENTER-TBI Investigators are provided in

Acknowledgements section.

Isabel R. A. Retel Helmrich

i.retelhelmrich@erasmusmc.nl

Extended author information available on the last page of the article $\begin{array}{ll}\text { TBI } & \text { Traumatic brain injury } \\ \text { QOLIBRI } & \text { Quality of Life after Brain Injury }\end{array}$

\section{Background}

Traumatic brain injury (TBI) is a leading cause of long-term impairments in functional, physical, mental, cognitive, and social domains [1]. These impairments are not restricted to severe cases, but are also known to occur frequently after moderate and mild TBI [2, 3]. Impairments can, for instance, be assessed using functional outcome scales [e.g., Glasgow Outcome Scale (Extended) (GOS(-E)] [4]. Although functional measurement scales are useful to portray functional problems, they do not capture the patient's subjective experience of their sequelae and wellbeing in daily life [5]. 
Therefore, there has been growing interest in HealthRelated Quality of Life (HRQoL) in TBI research. HRQoL focuses on an individuals' perception of how a disease and its treatments affect the physical, mental, and social aspects of their life [6]. Previous studies confirmed that long-term impairments following TBI affect (HR)QoL [7-16]. To assess HRQoL two types of instruments are available: generic and condition-specific instruments [6]. Generic instruments, such as the Short Form-36 (SF-36) [17], do not take into account diseases or particular conditions and allow comparison with healthy individuals, as well as various health states or conditions. It has been argued that generic HRQoL instruments may not be sensitive enough to detect key issues in TBI, such as cognitive dysfunctions and psychological issues $[6,18]$. A TBI-specific instrument, such as the Quality of Life after Traumatic Brain Injury (QOLIBRI) [19, 20], may, therefore, be complementary.

Outcomes following TBI depend on patient and injury characteristics, mechanisms of trauma, patient response, the social environment, and the quality of care provided [21-23]. Prognostic models predict the outcome of a patient based on characteristics at presentation and are important to help clinicians provide reliable information to patients and relatives [24]. It would be particularly helpful if poor HRQoL outcomes could be anticipated as these predictions could support clinicians in identifying patients who might benefit from close follow-up and early interventions. Although highquality and well-validated models exist to predict functional outcome following moderate and severe TBI [25], prognostic models for HRQoL following TBI have not been developed yet. Furthermore, efforts have been made to identify predictors of HRQoL following TBI [11, 12, 14, 26-31], but they are dispersed throughout the literature. Therefore, we aimed to identify predictors and develop prognostic models for HRQoL following mild, moderate, and severe TBI.

\section{Methods}

\section{Study population}

We analyzed patients included in the Collaborative European NeuroTrauma Effectiveness Research in Traumatic Brain Injury (CENTER-TBI, version Core 2.1) study. This is a prospective, multicenter, longitudinal, observational study $[32,33]$. Data were collected for patients with a clinical diagnosis of TBI and an indication for computed tomography (CT), presenting within $24 \mathrm{~h}$ of injury in one of the 58 participating centers. Participants were recruited from December 2014 to December 2017 in 18 countries across Europe and Israel.
For model development, patients were included if they were aged $\geq 18$ years and completed the SF-36v2 or QOLIBRI at 6 months post injury.

Data for the CENTER-TBI study were entered on the Quesgen e-CRF (Quesgen Systems, Inc., USA), hosted on the International Neuroinformatics Coordinating Facility (INCF) platform and extracted via the INCF Neurobot tool (INCF, Sweden). Informed consent was obtained from all participants according to local and national requirements.

\section{Candidate predictors}

Candidate predictors of HRQoL following TBI were selected based on literature, and included initial severity (Glasgow Come Scale) [12, 26, 27], age [28], sex [11, 28, 29], socioeconomic status [30], social support [28-31], pre-injury substance abuse [26, 28], and pre-injury mental health problems (e.g., anxiety, depression) [29, 34]. Additionally, major extracranial injury (MEI), injury cause, pre-injury health status, the presence of intracranial traumatic abnormalities, ongoing mental health problems, and 2 week HRQoL assessment were indicated by experts as potential predictors of HRQoL following TBI.

Ongoing mental health problems were assessed through scores for depression (PHQ9), anxiety (GAD7), and post-traumatic stress disorder (PCL5) at 2 weeks post injury. Socioeconomic status was assessed through type of education and type of employment. Social support was assessed through living arrangement. TBI severity was categorized into mild, moderate and severe based on the Glasgow Coma Scale (GCS) at admission. TBI was considered mild in patients with GCS 13-15, moderate in patients with GCS 9-12, and severe in patients with GCS of 3-8 [35]. MEI was defined as an Abbreviated Injury Scale (AIS) $\geq 3$ on any extracranial domain of the scale [36]. Pre-injury health status was assessed with the American Society of Anesthesiologists- physical status classification system (ASA-PS); patients are categorized as 'normal healthy patient', 'mild systemic disease', 'severe systemic disease', or 'severe systemic disease that is a constant threat to life'. The categories 'severe systemic disease' and 'severe systemic disease that is constant threat to life' were combined. The presence of intracranial traumatic abnormalities was assessed through the first computed tomography (CT) scan after injury, and indicates whether any of the 12 following abnormalities was present: mass lesion, hematoma, epidural hematoma, acute or subacute subdural hematoma, subdural collection mixed density, contusion, TAI, traumatic subarachnoid hemorrhage, intraventricular hemorrhage, midline shift or cisternal compression. The candidate predictors were assessed at admission within $24 \mathrm{~h}$, except for early HRQoL assessment and ongoing mental health problems, which were conducted 2 weeks post injury. 
Missing predictor values were imputed with 100 iterations with multiple imputation using the mice package [37]. All candidate predictors, injury severity score, and HRQoL outcomes between 2 weeks and 12 months were included in the imputation model.

\section{Outcome assessments}

The primary outcomes were the physical (PCS) and mental (MCS) component summary scores from the Short Form36v2 (SF-36v2) and the Quality of Life after Traumatic Brain Injury (QOLIBRI) total score at 6 months post injury. The SF-36v2 is a 36-item patient-reported outcome, which assesses multiple components of HRQoL: PCS; physical functioning, role limitations due to physical health, bodily pain, general health perceptions, vitality, MCS; social functioning, role limitations due to emotional health, and general mental health. Norm-based $T$-scores (standardized to mean 50 and SD of 10) were calculated for the PCS and MCS [17].

The QOLIBRI is a 37-item patient-reported outcome, consisting of four subscales assessing satisfaction with aspects of life (cognition, self, daily life and autonomy, and social relationships) and two subscales that concern how bothered the person is by difficulties (emotions, and physical problems) [19, 38].

\section{Data analyses}

Descriptive statistics were presented as medians [interquartile range (IQR)] or frequencies (percentage) for the predictors and HRQoL data. Differences in patient- and injury-related characteristics between responders, those who completed the SF-36v2 or QOLIBRI between 2 weeks and 12 months post injury, and non-responders were compared using independent sample $t$ tests (continuous) or $\chi^{2}$ tests (categorical).

We used linear regression analyses to quantify the relationship between predictors and the SF-36v2 PCS and MCS and the QOLIBRI total score at 6 months post injury. Model performance was expressed as proportion of variance explained $\left(R^{2}\right)$. For the continuous predictors-age and GCS - we assessed non-linearity with spline functions.

For each outcome, three prognostic models were defined: (I) the full model included all candidate predictors; (II) the extended model included a reduced set of predictors based on the Akaike information criteria (AIC); and (III) the core model included the three predictors with the largest partial $R^{2}$. We also explored the incremental value of HRQoL assessment and mental health problems at 2 weeks post injury for the prediction of the PCS, MCS and QOLIBRI total score. Incremental value was assessed by the difference in $R^{2}$ between the model with the additional predictors and the model without the additional predictors. Additionally, we explored the relationship between GCS (3-15) and all other predictors with interaction terms in multivariable analyses. Associations between predictors and outcome measures were presented with estimates of the regression coefficients and their $95 \%$ confidence interval (CI).

We assessed model performance through proportion explained variance $\left(R^{2}\right)$, and a bootstrapping procedure to reduce optimistic model performance estimates. Forty bootstrap samples were taken from the original dataset by sampling $X$ entries equal to the sample size of the original cohort with replacement. Performance of the model that was fitted on the bootstrap sample was evaluated both in the bootstrap sample and the original cohort and the difference indicated the optimism in performance [24].

Five sensitivity analyses were performed. First, the models were fitted for the PCS, MCS, and QOLIBRI total score for a subset of patients who completed the questionnaires individually or together with a relative, friend or caregiver, therefore, proxy responses were excluded. Second, the models were fitted for the PCS, MCS and QOLIBRI total score at 3 months rather than 6 months post injury. Third, instead of only selecting patients with available 6 months outcome, the models were also fitted with additional imputed 6 months outcome whenever 3 or 12 months outcomes were available. Fourth, analyses were performed in subgroups of TBI severity - mild versus moderate and severe. Fifth, the models were fitted for impaired SF-36 PCS and MCS $(<40)$ and QOLIBRI total scores $(<60)$ [39].

Analyses were performed with R statistical software 3.6.0 [40]. We used the rms package to fit the regression models [41]. Modeling results were reported in accordance with the TRIPOD guidelines [42].

\section{Results}

\section{Study population}

We included 2666 adult patients who completed the SF36v2 or the QOLIBRI between 2 weeks and 12 months post injury (Supplementary Fig. 1). Patients had a median age of 51 years (IQR $=33-65)$ (Table 1). More than half $(65 \%)$ of patients were male, and most (74\%) were diagnosed with mild TBI (GCS 13-15). A third (34\%) had major extracranial injury. More than half (53\%) were employed, and 24\% were retired. About $10 \%$ had pre-injury mental health problems. Moreover, less than half of the patients (42\%) experienced pre-injury comorbid health issues.

Responders and non-responders showed significant differences regarding baseline characteristics (Table 1). Nonresponders had a higher median age (47 vs. 51 years), and were more often male (71 vs. 65\%) (Table 1). Furthermore, they were more frequently diagnosed with moderate and 
Table 1 Patients' demographic and injury characteristics

\begin{tabular}{|c|c|c|c|}
\hline Characteristics & Responders $^{\mathrm{a}}(n=2666)$ & $\begin{array}{l}\text { Non-responders }{ }^{\mathrm{b}} \\
(n=1097)\end{array}$ & $p$-value* \\
\hline \multicolumn{4}{|l|}{ Demographics } \\
\hline Age (18-95) (median, [IQR]) & $51[33-65]$ & $47[30-65]$ & $>.05$ \\
\hline$\%$ Male sex & $65(1729)$ & $71(773)$ & $<.05$ \\
\hline \multicolumn{4}{|l|}{ Living arrangement $(N, \%)$} \\
\hline Together & $2093(79)$ & $834(76)$ & $<.05$ \\
\hline Missing (\%) & $3(0.1)$ & $8(1)$ & \\
\hline Highest level of education & & & $<.001$ \\
\hline None or primary school & $321(12)$ & $124(11)$ & \\
\hline Currently in or with diploma/degree oriented program & $555(21)$ & $199(18)$ & \\
\hline Secondary school/High school & $820(31)$ & $305(28)$ & \\
\hline College/University & $666(25)$ & $141(13)$ & \\
\hline Missing (\%) & $304(11)$ & $328(30)$ & \\
\hline Employment status & & & $<.001$ \\
\hline Yes & $1410(53)$ & $453(41)$ & \\
\hline No & 447 (17) & $210(19)$ & \\
\hline Retired & $643(24)$ & $243(22)$ & \\
\hline Missing (\%) & $166(6)$ & $191(17)$ & \\
\hline Employment type $(N, \%)$ & & & $<.001$ \\
\hline Working & $1410(53)$ & $453(41)$ & \\
\hline Looking for work, unemployed & $145(5)$ & $74(7)$ & \\
\hline Unable to work/sick leave & $70(3)$ & $39(4)$ & \\
\hline Retired & $643(24)$ & $243(22)$ & \\
\hline Student & $190(7)$ & $74(7)$ & \\
\hline Homemaker & $42(2)$ & $23(2)$ & \\
\hline Missing (\%) & $166(6)$ & $191(18)$ & \\
\hline \multicolumn{4}{|l|}{ Pre-injury health status } \\
\hline Pre-injury ASA-PS classification & & & $<.001$ \\
\hline Normal healthy patient & $1527(57)$ & $592(57)$ & \\
\hline Mild systemic disease & $872(33)$ & $334(30)$ & \\
\hline Severe systemic disease & $233(9)$ & $115(11)$ & \\
\hline Missing (\%) & $34(1)$ & $56(5)$ & \\
\hline History of substance abuse ${ }^{c}$ & & & $<.001$ \\
\hline Yes & $72(3)$ & $58(5)$ & \\
\hline Missing (\%) & $43(2)$ & $59(5)$ & \\
\hline Pre-injury mental health problems ${ }^{\mathrm{d}}$ & & & $<.001$ \\
\hline Yes & $268(10)$ & $124(11)$ & \\
\hline Missing (\%) & $43(2)$ & $59(5)$ & \\
\hline \multicolumn{4}{|l|}{ Injury characteristics } \\
\hline Cause of Injury & & & $<.001$ \\
\hline Road traffic accident & $1041(39)$ & $371(34)$ & \\
\hline Incidental fall & $1187(45)$ & $486(44)$ & \\
\hline Other non-intentional injury & $239(9)$ & $84(8)$ & \\
\hline Violence or assault & $125(5)$ & $99(9)$ & \\
\hline Suicide attempt & $22(1)$ & $13(1)$ & \\
\hline Missing (\%) & $52(2)$ & $44(4)$ & \\
\hline GCS (3-15) & & & $<.001$ \\
\hline Mild (13-15) & $1981(74)$ & $713(65)$ & \\
\hline Moderate/Severe (3-12) & $605(23)$ & $338(31)$ & \\
\hline Missing & $80(3)$ & $46(4)$ & \\
\hline
\end{tabular}


Table 1 (continued)

\begin{tabular}{|c|c|c|c|}
\hline Characteristics & Responders $^{\mathrm{a}}(n=2666)$ & $\begin{array}{l}\text { Non-responders }{ }^{\mathrm{b}} \\
(n=1097)\end{array}$ & $p$-value* \\
\hline ISS (0-75) (Median, [IQR]) & $13[8-25]$ & $16[9-28]$ & $<.001$ \\
\hline Missing (\%) & $34(1)$ & $17(1)$ & \\
\hline Intracranial traumatic abnormalities (present) & $1381(52)$ & $555(51)$ & \\
\hline Missing (\%) & $168(6)$ & $132(12)$ & \\
\hline $\mathrm{MEI}^{\mathrm{e}}$ & & & $>.05$ \\
\hline Yes & $909(34)$ & $410(37)$ & \\
\hline Total percentage of observations of baseline characteristics missing & 3 & 7 & \\
\hline \multicolumn{4}{|l|}{ Mental health problems 2 weeks post injury $(N=609)$} \\
\hline Depression $(0-27)$ & $5[1-10]$ & NA & \\
\hline Missing (\%) & $77(2054)$ & & \\
\hline Anxiety $(0-21)$ & $2[0-6]$ & NA & \\
\hline Missing (\%) & $77(2054)$ & & \\
\hline Post-traumatic stress disorder $(0-72)$ & 9 [3-19] & NA & \\
\hline Missing (\%) & $77(2057)$ & & \\
\hline
\end{tabular}

AIS Abbreviated Injury Scale, ASA-PS The American Society of Anesthesiologists-physical status classification system, GCS Glasgow Coma Scale, ISS Injury Severity Score, $N$ number, $M E I$ major extracranial injury, $S D$ standard deviation

$* p$-values from ANOVA for continuous and $\chi^{2}$ statistics for categorical variables

${ }^{\text {a Patients }}<18$ years of age $(N=158)$ and non-responders $(N=1588)$ were excluded

${ }^{\text {b}}$ Patients $<18$ years of age $(N=108)$ and deceased patients $(N=491)$ were excluded

${ }^{c}$ Patients with a history of substance abuse disorder prior to the injury

${ }^{\mathrm{d}}$ Patients with a history of anxiety, depression, sleep disorders, or schizophrenia prior to the injury

${ }^{\mathrm{e}}$ Patients with an Abbreviated Injury Scale $\geq 3$ regarding the following body regions; face, cervical spine, thorax/chest, abdomen/pelvic contents, extremities and pelvic girdle, or external (skin), thus excluding head and neck

severe TBI than responders, and had higher median injury severity score (16 vs. 13).

The median PCS, MCS and QOLIBRI total scores increased between 3 and 12 months post injury. The largest improvements were observed between 3 and 6 months (Fig. 1; Supplementary Table 1). PCS showed larger improvements than MCS in patients after mild as well as patients after moderate and severe TBI. At 6 months, 23\% of patients after mild and $33 \%$ of patients after moderate and severe TBI fell within the 'impaired' category on the PCS. On the MCS, 26\% of patients after mild and 33\% of patients after moderate and severe TBI had impaired HRQoL, and on the QOLIBRI 22\% of patients after mild and $34 \%$ of patients after moderate and severe TBI classified as 'impaired' 6 months post injury. As expected, patients after moderate and severe TBI had lower median HRQoL scores than patients after mild TBI at every time point. The MCS and QOLIBRI (spearman 0.73) were more strongly related than with PCS (spearman 0.26 with MCS and 0.57 with QOLIBRI; Supplementary Fig. 2).

\section{Model development}

For the predictor values most (97\%) observations were complete. Of the predictors with the highest percentage missing, $89 \%$ and $94 \%$ of observations were complete (Table 1).

\section{Physical health component summary score}

The strongest predictors of PCS 6 months after TBI were GCS, MEI, and pre-injury health status (ASA-PS) (Table 2; Fig. 2). We found no significant interactions between GCS and the other candidate predictors $(p>0.05)$, indicating that predictors of PCS did not differ between patients with mild (GCS $\geq 13$ ), and moderate and severe TBI (GCS $\leq 12$ ). Severe systemic disease had a strong prognostic effect, indicating that patients with severe pre-injury comorbidities had lower PCS 6 months post injury (Table 2; Supplementary Fig. 3). The model had an $R^{2}$ of $11 \%$ when the three strongest predictors were considered in the core model. The extended model, also including age, sex, type of employment, and level of education, performed notably better $\left(R^{2}=19 \%\right)$. 


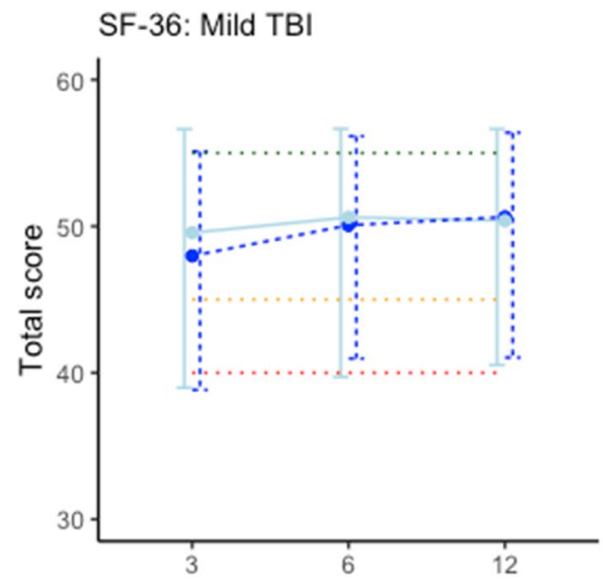

QOLIBRI: Mild TBI

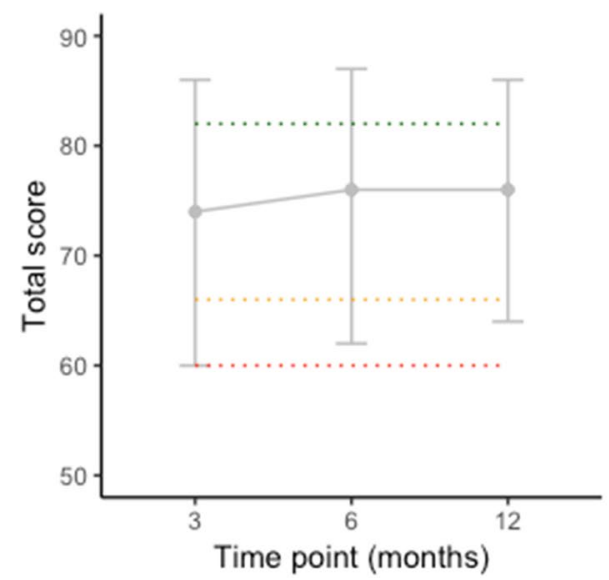

Fig. 1 Plots of the median SF-36v2 physical and mental health component summary scores (top) and the Quality of Life after Traumatic Brain Injury (bottom) by time point for mild (left), and moderate and severe TBI (right). For the SF-36v2, scores of 45-55 are considered within the average range (green/upper dotted line), scores of 40-45 are considered borderline (orange/middle dotted line), and scores

\section{Mental health component summary score}

The strongest predictors of MCS 6 months after TBI were pre-injury mental health problems, level of education, and type of employment (Table 3; Fig. 2). Again, we found no significant interactions between GCS and the other candidate predictors $(p>0.05)$. Patients with a low level of education, as well as those who are unemployed, unable to work, or homemakers had lower MCS 6 months after injury (Table 3; Supplementary Fig. 3). The model had an $R^{2}$ of $6 \%$ when the three strongest predictors, pre-injury mental health problems, level of education and type of employment, were considered in the core model. The extended model, also including age, employment, education and sex, performed somewhat better $\left(R^{2}=9 \%\right)$.
SF-36: Moderate and severe TBI

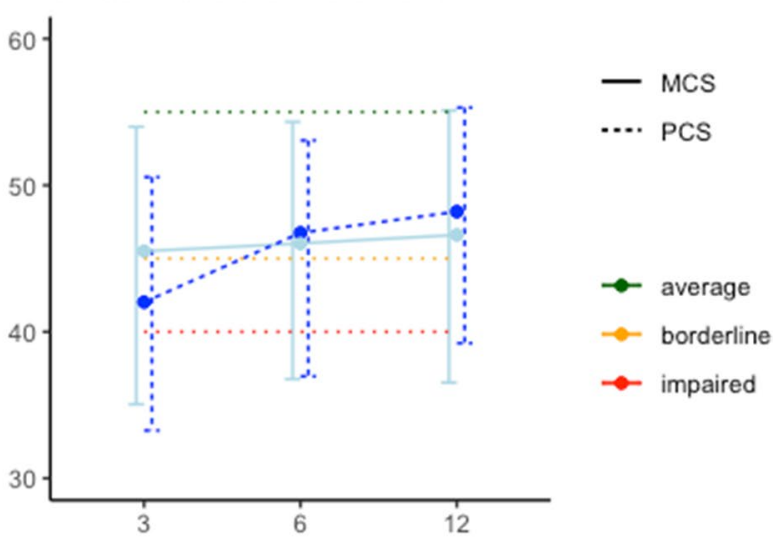

QOLIBRI: Moderate and severe TBI

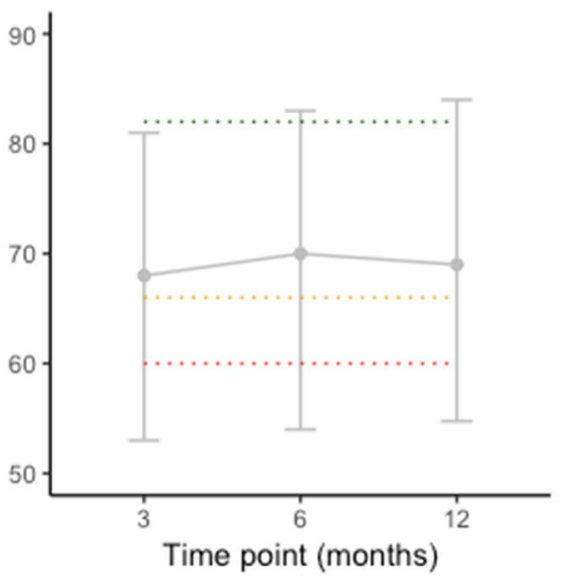

- QOLIBRI

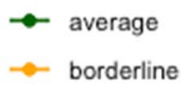

- impaired

below 40 (red/lower dotted line) are considered impaired (Ware et al. 2007). For the QOLIBRI, scores of 67-82 are considered within the average range (green/upper dotted line), scores of 60-66 are considered borderline (orange/middle dotted line), and scores below 60 (red/lower dotted line) are considered impaired (Wilson et al. 2017). (Color figure online)

\section{Quality of Life after Traumatic Brain Injury total score}

The strongest predictors of the QOLIBRI total score at 6 months were type of employment, level of education and pre-injury mental health problems (Table 4; Fig. 2), which was similar to the MCS. Again, we found no significant interactions between GCS and the other candidate predictors $(p>0.05)$. Model performance for the QOLIBRI was intermediate to that of the models for PCS and MCS $\left(R^{2}\right.$ $13 \%$, compared to $18 \%$ for PCS and $9 \%$ for MCS full models) (Table 4). 
Table 2 Regression coefficients and 95\% confidence intervals for the SF-36v2 physical health component summary score (PCS) with multivariable linear regression analysis

\begin{tabular}{|c|c|c|c|}
\hline PCS & Core Model & Extended Model & Full Model \\
\hline Constant & 46 & 49 & 49 \\
\hline \multicolumn{4}{|l|}{ Predictors } \\
\hline GCS & $0.35(0.25,0.46)$ & $0.38(0.28,0.49)$ & $0.39(0.28,0.49)$ \\
\hline \multicolumn{4}{|l|}{$\mathrm{MEI}\left(\mathrm{No}^{\mathrm{a}}\right)$} \\
\hline Yes & $-3.7(-4.6,-2.8)$ & $-4.2(-5.1,-3.3)$ & $-4.1(-5.0,-3.1)$ \\
\hline \multicolumn{4}{|l|}{ ASA-PS (Healthy patient ${ }^{\mathrm{a}}$ ) } \\
\hline Mild systemic disease & $-4.0(-5.0,-3.1)$ & $-2.0(-3.0,-1.0)$ & $-2.0(-3.0,-0.96)$ \\
\hline Severe systemic disease & $-10.0(-12.0,-8.9)$ & $-7.2(-8.8,-5.5)$ & $-7.3(-9.0,-5.7)$ \\
\hline \multicolumn{4}{|l|}{ Education (College/Uni degree ${ }^{\mathrm{a}}$ ) } \\
\hline Currently in school & & $-1.7(-2.9,-0.51)$ & $-1.8(-3.0,-0.60)$ \\
\hline None/Primary school & & $-4.3(-5.8,-2.8)$ & $-4.3(-5.8,-2.8)$ \\
\hline Secondary/high school & & $-1.5(-2.6,-0.38)$ & $-1.6(-2.7,-0.45)$ \\
\hline \multicolumn{4}{|l|}{ Employment (Working ${ }^{\mathrm{a}}$ ) } \\
\hline Homemaker & & $-4.4(-8.2,-0.55)$ & $-4.6(-8.5,-0.81)$ \\
\hline Student & & $0.41(-1.4,2.2)$ & $0.45(-1.4,2.3)$ \\
\hline Retired & & $-1.3(-2.7,0.10)$ & $-1.4(-2.8,-0.06)$ \\
\hline Unable to work/sick leave & & $-6.3(-8.8,-3.7)$ & $-6.1(-8.8,-3.5)$ \\
\hline Unemployed & & $-3.2(-5.1,-1.2)$ & $-3.0(-5.0,-1.0)$ \\
\hline Age (per decade) & & $-0.73(-1.0,-0.36)$ & $-0.74(-1.1,-0.36)$ \\
\hline \multicolumn{4}{|l|}{ Sex $\left(\right.$ Male $\left.^{a}\right)$} \\
\hline Female & & $-2.1(-3.0,-1.2)$ & $-2.0(-2.9,-1.1)$ \\
\hline \multicolumn{4}{|l|}{ Injury cause (Road traffic ${ }^{a}$ ) } \\
\hline Incidental fall & & & $0.71(-0.24,1.7)$ \\
\hline Other non-intentional injury & & & $-0.50(-1.1,2.1)$ \\
\hline Violence/Assault & & & $-0.18(-2.0,2.3)$ \\
\hline Suicide attempt & & & $-1.4(-5.8,2.9)$ \\
\hline \multicolumn{4}{|l|}{ Pre-injury substance abuse $\left(\mathrm{No}^{\mathrm{a}}\right)$} \\
\hline Yes & & & $3.2(0.43,6.0)$ \\
\hline \multicolumn{4}{|c|}{ Pre-injury mental health problems $\left(\mathrm{No}^{\mathrm{a}}\right)$} \\
\hline Yes & & & $-1.2(-2.6,0.26)$ \\
\hline \multicolumn{4}{|l|}{ Living arrangement (Together ${ }^{\mathrm{a}}$ ) } \\
\hline Alone & & & $-0.87(-1.9,0.16)$ \\
\hline$R^{2}$ development cohort & 0.13 & 0.20 & 0.21 \\
\hline Optimism & $0.01^{\mathrm{b}}$ & 0.01 & 0.02 \\
\hline$R^{2}$ after bootstrap validation & - & 0.19 & 0.19 \\
\hline
\end{tabular}

Model performance indicated by explained variance $\left(R^{2}\right)$ and bootstrap validation for each model $(N=2073)$

${ }^{a}$ Reference category of categorical variable

${ }^{\mathrm{b}}$ Optimism of the core model is estimated to be similar to that of the extended model

Core model Glasgow Coma Scale, Major extracranial injury and pre-injury health status (ASA-PS), Extended model core plus education, employment, age and sex, Full model extended plus injury cause, pre-injury substance abuse, pre-injury mental health problems, and living arrangement

\section{Early HRQoL assessment, ongoing mental health and intracranial lesions}

In a subgroup of patients following predominantly mild TBI (99\%), early HRQoL assessment at 2 weeks (SF-36v2 $N=432$ and QOLIBRI $N=434$ ) had substantial incremental value (PCS $R^{2} 37 \%$ compared to $15 \%$ of the full model without 2 week PCS; MCS $36 \%$ compared to $12 \%$ of the full model without 2 week MCS; QOLIBRI 48\% compared to $10 \%$ of the full model without 2 week QOLIBRI) (Fig. 3). Similarly, depression, anxiety, and PTSD at 2 weeks (SF$36 \mathrm{v} 2 N=418$ and QOLIBRI $N=420$ ) had substantial incremental value for the prediction of MCS and the QOLIBRI (MCS $R^{2}=35 \%$ compared to $11 \%$ of the full model without 


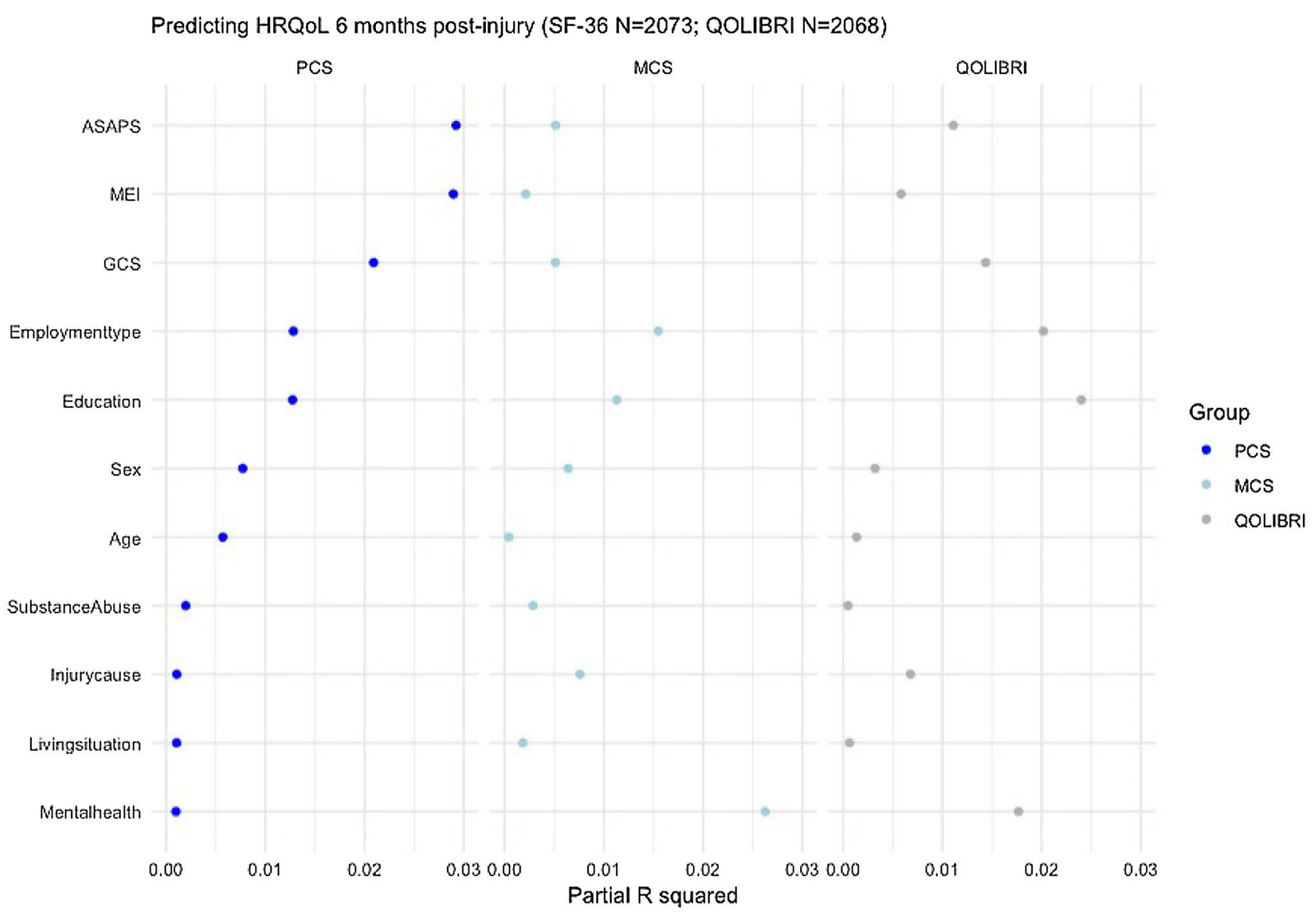

Fig. 2 Contribution of predictors to partial explained variance $\left(R^{2}\right)$ of the models for PCS (left), MCS (middle), and QOLIBRI (right). The partial $R^{2}$ is calculated as follows: Total $R^{2}$ of multivariable

2 week depression, anxiety and PTSD; QOLIBRI $=37 \%$ compared to $12 \%$ of the full model without 2 week depression, anxiety and PTSD). However, the addition of mental health problems 2 weeks post injury had limited incremental value for the prediction of PCS (PCS $R^{2}=22 \%$ compared to $16 \%$ of the full model without 2 week depression, anxiety and PTSD). Furthermore, for the prediction of PCS, MCS and the QOLIBRI, the addition of intracranial traumatic abnormalities ( $N=1642$ and 1639) had no or limited incremental value (PCS $R^{2}=20 \%$ compared to $19 \%$ of the full model without intracranial traumatic abnormalities; MCS $10 \%$ compared to $10 \%$; QOLIBRI $14 \%$ compared to $13 \%$ ).

\section{Sensitivity analyses}

Model performance was similar when proxy responses (PCS and MCS $N=98$, QOLIBRI $N=93$ ) were excluded. The full models also performed similarly when 3 month rather than 6 month HRQoL was predicted (PCS $R^{2} 20 \%$ model $-R^{2}$ multivariable model without individual predictor/Total $R^{2}$ of multivariable model without individual predictor $=$ Partial $R^{2}$

vs $19 \%$ when the model was fitted for 6 month outcome, respectively; MCS $R^{2} 9 \%$ vs $9 \%$; QOLIBRI $R^{2} 14 \%$ vs $13 \%$;). Furthermore, the models performed similarly when missing 6 month HRQoL outcomes $(N=462)$ were imputed for with HRQoL outcomes on 3 and 12 months (PCS $R^{2} 20 \%$ vs $19 \%$, respectively; MCS $R^{2} 9 \%$ vs $9 \%$; QOLIBRI $R^{2} 13 \%$ vs $13 \%$ ) (Supplementary Tables 2, 3, $4)$. As expected, the predictive value of GCS diminished when patients were separated based on GCS (Mild $\geq 13$, Moderate and Severe $\leq 12$ ) (Supplementary Fig. 4). The models were fitted for impaired PCS and MCS $(<40)$ and QOLIBRI total scores $(<60)$. The strongest predictors 
Table 3 Regression coefficients and $95 \%$ confidence intervals for the SF-36v2 mental health component summary score (MCS) with multivariable linear regression analysis

\begin{tabular}{|c|c|c|c|}
\hline MCS & Core Model & Extended Model & Full Model \\
\hline Constant & 49 & 45 & 44 \\
\hline \multicolumn{4}{|l|}{ Predictors } \\
\hline \multicolumn{4}{|c|}{ Pre-injury mental health problems $\left(\mathrm{No}^{\mathrm{a}}\right)$} \\
\hline Yes & $-7.5(-9.2,-5.9)$ & $-6.9(-8.6,-5.1)$ & $-6.8(-8.6,-5.1)$ \\
\hline \multicolumn{4}{|l|}{ Education (College/Uni degree ${ }^{\mathrm{a}}$ ) } \\
\hline Currently in school & $-1.7(-3.2,-0.28)$ & $-1.8(-3.3,-0.40)$ & $-1.8(-3.3,-0.39)$ \\
\hline None/Primary school & $-4.4(-6.1,-2.6)$ & $-4.3(-6.1,-2.6)$ & $-4.4(-6.1,-2.6)$ \\
\hline Secondary/high school & $-0.96(-2.3,0.36)$ & $-0.85(-2.2,0.46)$ & $-0.84(-2.1,0.47)$ \\
\hline \multicolumn{4}{|l|}{ Employment (Working ${ }^{\mathrm{a}}$ ) } \\
\hline Homemaker & $-6.4(-11.0,-1.9)$ & $-4.4(-8.9,0.12)$ & $-4.5(-9.1,0.07)$ \\
\hline Student & $-0.33(-2.3,1.6)$ & $-0.48(-2.4,1.5)$ & $-0.31(-2.5,1.9)$ \\
\hline Retired & $2.1(0.87,3.3)$ & $2.5(1.1,3.8)$ & $2.3(0.60,4.0)$ \\
\hline Unable to work/sick leave & $-5.8(-8.9,-2.7)$ & $-4.5(-7.6,-1.4)$ & $-4.6(-7.7,-1.5)$ \\
\hline Unemployed & $-4.1(-6.5,-1.7)$ & $-4.0(-6.4,-1.6)$ & $-4.0(-6.4,-1.6)$ \\
\hline \multicolumn{4}{|l|}{ Injury cause (Road traffic ${ }^{\mathrm{a}}$ ) } \\
\hline Incidental fall & & $2.2(1.1,3.4)$ & $2.2(1.1,3.3)$ \\
\hline Other non-intentional injury & & $1.2(-0.67,3.1)$ & $1.2(-0.69,3.1)$ \\
\hline Violence or Assault & & $0.01(-2.6,2.6)$ & $-0.04(-2.5,2.6)$ \\
\hline Suicide attempt & & $4.9(-0.15,10.0)$ & $4.9(-0.15,10.0)$ \\
\hline GCS & & $0.22(0.10,0.34)$ & $0.22(0.09,0.34)$ \\
\hline \multicolumn{4}{|l|}{ ASA-PS (Healthy patient ${ }^{\mathrm{a}}$ ) } \\
\hline Mild systemic disease & & $-0.95(-2.1,0.21)$ & $-1.0(-2.2,0.20)$ \\
\hline Severe systemic disease & & $-3.4(-5.4,-1.5)$ & $-3.5(-5.5,-1.5)$ \\
\hline \multicolumn{4}{|l|}{ Pre-injury substance abuse $\left(\mathrm{No}^{\mathrm{a}}\right)$} \\
\hline Yes & & $-4.4(-7.7,-1.1)$ & $-4.3(-7.6,-1.0)$ \\
\hline \multicolumn{4}{|l|}{ Sex $\left(\right.$ Male $\left.^{\mathrm{a}}\right)$} \\
\hline Female & & $-2.1(-3.2,-1.0)$ & $-2.1(-3.2,-1.0)$ \\
\hline \multicolumn{4}{|l|}{ Living arrangement (Together ${ }^{\mathrm{a}}$ ) } \\
\hline Alone & & $-1.3(-2.5,0.06)$ & $-1.3(-2.5,-0.07)$ \\
\hline \multicolumn{4}{|l|}{$\mathrm{MEI}\left(\mathrm{No}^{\mathrm{a}}\right)$} \\
\hline Yes & & $-1.2(-2.3,-0.15)$ & $-1.2(-2.3,-0.15)$ \\
\hline Age (per decade) & & & $0.08(-0.37,0.53)$ \\
\hline$R^{2}$ development cohort & 0.08 & 0.11 & 0.11 \\
\hline$R^{2}$ optimism & $0.02^{\mathrm{b}}$ & 0.02 & 0.02 \\
\hline$R^{2}$ after bootstrap validation & - & 0.09 & 0.09 \\
\hline
\end{tabular}

Model performance indicated by explained variance $\left(R^{2}\right)$ and bootstrap validation for each model $(N=2073)$

Core model pre-injury mental health problems, education and employment, Extended model core plus injury cause, GCS, ASA-PS, living arrangement, MEI and sex, Full model extended plus age

${ }^{a}$ Reference category of categorical variable

${ }^{\mathrm{b}}$ Optimism of the core model is estimated to be similar to that of the extended model 
Table 4 Regression coefficients and 95\% confidence intervals for the Quality of Life after Traumatic Brain Injury (QOLIBRI) total score with multivariable linear regression analysis

\begin{tabular}{|c|c|c|c|}
\hline QOLIBRI & Core Model & Extended Model & Full Model \\
\hline Constant & 78 & 70 & 73 \\
\hline \multicolumn{4}{|l|}{ Predictors } \\
\hline \multicolumn{4}{|c|}{ Pre-injury mental health problems $\left(\mathrm{No}^{\mathrm{a}}\right)$} \\
\hline Yes & $-9.8(-12.0,-7.2)$ & $-9.0(-12.0,-6.3)$ & $-8.8(-11.0,-6.2)$ \\
\hline \multicolumn{4}{|l|}{ Education (College/Uni degree ${ }^{\mathrm{a}}$ ) } \\
\hline Currently in school & $-5.1(-7.3,-2.8)$ & $-5.0(-7.2,-2.8)$ & $-5.0(-7.2,-2.8)$ \\
\hline None/Primary school & $-11.0(-14.0,-8.0)$ & $-10.0(-13.0,-7.6)$ & $-10.0(-13.0,-7.4)$ \\
\hline Secondary/high school & $-4.8(-6.9,-2.8)$ & $-4.4(-6.4,-2.4)$ & $-4.5(-6.5,-2.5)$ \\
\hline \multicolumn{4}{|l|}{ Employment (Workinga) } \\
\hline Homemaker & $-12.0(-19.0,-5.6)$ & $-10.0(-17.0,-3.1)$ & $-9.1(-16.0,-2.2)$ \\
\hline Student & $-1.6(-1.5,4.6)$ & $-1.3(-1.7,4.3)$ & $-0.11(-3.5,3.2)$ \\
\hline Retired & $-0.30(-2.2,1.6)$ & $-0.47(-1.6,2.5)$ & $2.0(-0.62,4.6)$ \\
\hline Unable to work/sick leave & $-11.0(-16.0,-6.4)$ & $-9.4(-14.0,-4.8)$ & $-8.6(-13.0,-3.9)$ \\
\hline Unemployed & $-9.4(-13.0,-5.7)$ & $-9.1(-13.0,-5.4)$ & $-9.2(-13.0,-5.5)$ \\
\hline \multicolumn{4}{|l|}{ Injury cause (Road traffic ${ }^{a}$ ) } \\
\hline Incidental fall & & $2.8(1.1,4.6)$ & $3.1(1.4,4.9)$ \\
\hline Other non-intentional injury & & $3.2(0.32,6.0)$ & $3.3(0.43,6.1)$ \\
\hline Violence or Assault & & $-1.0(-5.0,3.0)$ & $-1.2(-5.2,2.8)$ \\
\hline Suicide attempt & & $3.1(-4.8,11.0)$ & $3.2(-4.7,11.0)$ \\
\hline GCS & & $0.56(0.37,0.74)$ & $0.57(0.38,0.76)$ \\
\hline \multicolumn{4}{|l|}{ ASA-PS (Healthy patient ${ }^{\mathrm{a}}$ ) } \\
\hline Mild systemic disease & & $-2.4(-4.2,-0.66)$ & $-1.9(-3.8,0.09)$ \\
\hline Severe systemic disease & & $-8.9(-12.0,-5.8)$ & $-8.1(-11.0,-5.0)$ \\
\hline \multicolumn{4}{|l|}{ Pre-injury substance abuse $\left(\mathrm{No}^{\mathrm{a}}\right)$} \\
\hline Yes & & & $-2.9(-8.3,2.4)$ \\
\hline \multicolumn{4}{|l|}{ Sex $\left(\right.$ Male $\left.^{a}\right)$} \\
\hline Female & & $2.4(0.74,4.0)$ & $-2.3(-4.0,-0.69)$ \\
\hline \multicolumn{4}{|l|}{ Living arrangement (Together $\left.{ }^{\mathrm{a}}\right)$} \\
\hline Alone & & & $-1.2(-3.1,0.68)$ \\
\hline \multicolumn{4}{|l|}{$\mathrm{MEI}\left(\mathrm{No}^{\mathrm{a}}\right)$} \\
\hline Yes & & $-3.1(-4.8,-1.4)$ & $-3.2(-4.9,-1.5)$ \\
\hline Age (per decade) & & $-0.62(-1.3,0.07)$ & $-0.63(-1.3,0.06)$ \\
\hline$R^{2}$ development cohort & 0.10 & 0.15 & 0.15 \\
\hline$R^{2}$ optimism & $0.02^{\mathrm{b}}$ & 0.02 & 0.02 \\
\hline$R^{2}$ after bootstrap validation & - & 0.13 & 0.13 \\
\hline
\end{tabular}

Model performance indicated by explained variance $\left(R^{2}\right)$ and bootstrap validation for each model $(N=2068)$

Core model Education, employment type and pre-injury mental health problems, Extended model core plus injury cause, GCS, ASA-PS, sex, MEI, and age, Full model extended plus pre-injury substance abuse, and living arrangement

${ }^{a}$ Reference category of categorical variable

${ }^{\mathrm{b}}$ Optimism of the core model is estimated to be similar to that of the extended model 
Including HRQoL at 2 weeks (SF-36 N=432; QOLIBRI N=434)

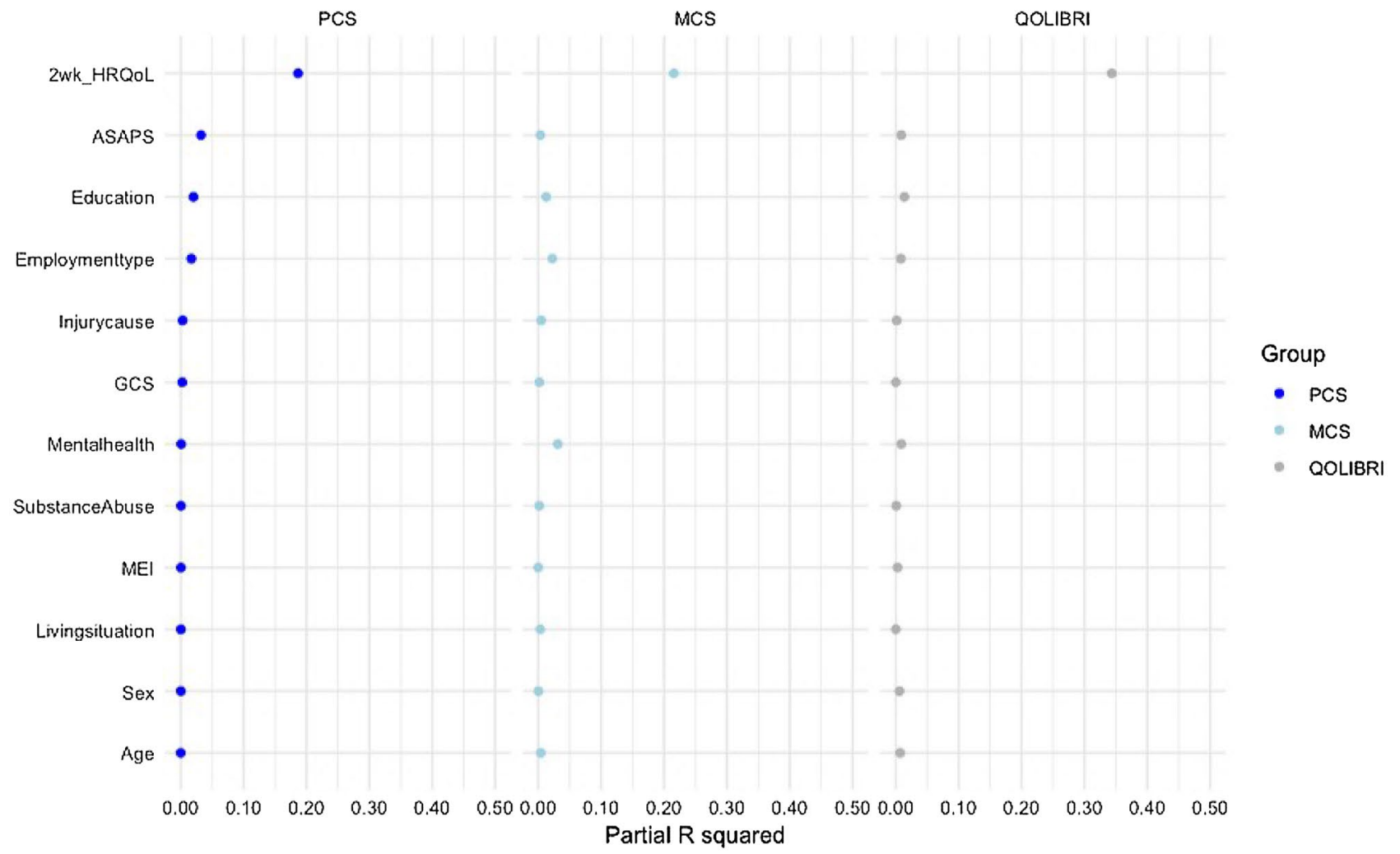

Fig. 3 Contribution of predictors to partial explained variance $\left(R^{2}\right)$ of the full models for PCS (left), MCS (middle), and QOLIBRI (right) including early HRQoL assessment at 2 weeks

of impaired PCS were GCS, pre-injury health status and MEI (Supplementary Table 5). For impaired MCS the strongest predictors were pre-injury mental health problems, employment type and level of education (Supplementary Table 6). The strongest predictors of impaired QOLIBRI total score were GCS, level of education, and employment type (Supplementary Table 7).

\section{Model presentation}

The proposed models were presented with nomograms (Supplementary Figs. 5, 6, 7). Prognostic HRQoL scores at 6 months post injury can be calculated for individual patients using the formulas (Textbox 1; Supplementary Table 8).

Textbox 1 Example of calculation of individual SF-36v2 physical (PCS) and mental (MCS) component summary scores and the Quality of Life after Traumatic Brain Injury (QOLIBRI) total score at 6 months post injury based on the core models

\begin{tabular}{lllll}
\hline & $\begin{array}{l}\text { Patient } \\
\text { character- } \\
\text { istics }\end{array}$ & $\begin{array}{l}\text { PCS score } \\
(T \text {-scores })\end{array}$ & $\begin{array}{l}\text { MCS score } \\
(T \text {-scores })\end{array}$ & $\begin{array}{l}\text { QOLIBRI } \\
\text { score } \\
(0-100)\end{array}$ \\
\hline Constant & & 46 & 49 & 78 \\
GCS & 13 & $0.35 \times 13$ & & \\
MEI & Yes & $-3.7 \times 1$ & & \\
ASA-PS & Mild & $-4.0 \times 1$ & & \\
& $\begin{array}{l}\text { systemic } \\
\text { disease }\end{array}$ & & $-7.5 \times 1$ & $-9.8 \times 1$ \\
$\begin{array}{c}\text { Pre-injury } \\
\text { mental } \\
\text { health } \\
\text { problems }\end{array}$ & Yes & & & \\
$\begin{array}{c}\text { Education } \\
\text { level }\end{array}$ & High school & & $-0.96 \times 1$ & $-4.8 \times 1$ \\
$\begin{array}{c}\text { Employ- } \\
\text { ment type }\end{array}$ & Retired & & $2.1 \times 1$ & $-0.30 \times 1$ \\
$\begin{array}{c}\text { Sum score } \\
\text { Mebl }\end{array}$ & & 43 & 43 & 63 \\
\hline
\end{tabular}




\section{Discussion}

We developed simple and more extended models for predicting Health-Related Quality of Life (HRQoL) 6 months after traumatic brain injury (TBI), separately for the SF36v2 physical (PCS) and mental component summary scores (MCS) and the Quality of Life after Traumatic Brain Injury (QOLIBRI) total score. Medical and injury-related characteristics were most important for the prediction of PCS, whereas patient-related characteristics were more relevant for prediction of MCS and the QOLIBRI. Moderate model performance is indicative for the complexity of predicting HRQoL. Substantial improvement in model performance was achieved by including 2-week HRQoL assessment.

Although previously indicated predictors of HRQoL following TBI were also relevant in our study the proportion explained variance $\left(R^{2}\right)$ of the models was moderate. Models that include predictors that move beyond baseline assessment, also known as dynamic or longitudinal predictors, have been proposed to update existing models and potentially improve performance [21]. Prior studies have shown the importance of aspects of current status, including emotional state, for the prediction of HRQoL following TBI [14, 38, 43]. As expected, our study demonstrated that early HRQoL assessment substantially improved model performance in a subset of patients with predominantly mild TBI; the $R^{2}$ for PCS was $38 \%$ compared to $17 \%$ of the full model without 2 week HRQoL; for MCS the increase was to $35 \%$ from $12 \%$, and for the QOLIBRI the $R^{2}$ increased from 19 to $54 \%$. In our study, HRQoL was highly variable between TBI patients over time, whereas within patients HRQoL scores might be more stable. This could explain the substantial incremental value of 2 week HRQoL for the prediction of 6 month HRQoL outcomes. In our study, adherence varied across time points; 2 week HRQoL assessment was only available in patients that were seen in the Emergency Room (ER) and discharged or in the hospital ward other than the ICU, which almost exclusively comprised mild TBI patients (99\%) without MEI (91\%). Therefore, the incremental value of early HRQoL assessment can only be generalized to patients following mild TBI. Early after injury, patients might be unable or less inclined to respond to questionnaires. Although patient-reported outcomes are increasingly reported in clinical practice, variable or low adherence over time makes early follow-up assessments less feasible to collect, which limits the clinical applicability of dynamic prediction models using patient-reported outcomes or assessments. Other longitudinal predictors that can be considered to be included for the prediction of HRQoL following TBI that may be less dependent on patient response are, for instance, biomarkers, duration of hospital stay, and length of coma.
In our study, most patients (74\%) classified as mild TBI. More than half $(1381 / 2666,52 \%)$ had intracranial traumatic abnormalities on the initial computed tomography (CT) scan, which might be related to worse long-term outcome and lower HRQoL. In patients following mild TBI, the presence or absence of intracranial traumatic abnormalities is used to differentiate between complicated and uncomplicated mild TBI [44]. A recent study found that although patients after complicated mild TBI reported slightly more post-concussion symptoms compared to those after uncomplicated mild TBI, an abnormality on initial CT was only a weak indicator of these problems after adjusting for baseline covariates (e.g., age, gender, GCS) [45]. However, the relationship between intracranial traumatic abnormalities and HRQoL following TBI has not been examined yet. Our study indicates that when adjusting for patient- and injury-related characteristics, intracranial traumatic abnormalities had limited to no incremental value for the prediction of HRQoL following TBI. As intracranial traumatic abnormalities are relevant to address the heterogeneity in patients following mild TBI [44], a formal investigation of the relationship between intracranial traumatic abnormalities and HRQoL in a subgroup of patients following mild TBI is warranted. A recent study indicates that the Helsinki CT classification was associated with QoL up to 4 years after TBI [46]. Besides the presence of intracranial traumatic abnormalities, more detailed information such as CT lesion phenotypes, their location, extent and clustering could therefore be considered.

TBI can lead to long-term impairments in functional, physical, mental, cognitive, and social domains. Although median MCS was initially higher than PCS at 3 months, PCS showed greater improvements between 6 and 12 months post injury. This indicates that over time mental health was more strongly affected by TBI. These findings also advocate for a multidimensional outcome assessment of TBI that captures a broad range of difficulties patients may experience, including physical, psychosocial and emotional outcomes. Furthermore, prior studies have shown that patients who sustained TBI, on average, show large HRQoL deficits from full recovery after 12 months when measured by population norms [6]. In our study, post hoc analyses confirmed these findings in mild as well as moderate and severe TBI patients; at 12 months $22 \%$ of mild and $27 \%$ of moderate and severe TBI patients had impaired PCS scores. Similarly, 24\% of mild and $35 \%$ of moderate and severe TBI patients had impaired MCS scores, and $21 \%$ of mild and $33 \%$ of moderate and severe TBI patients had impaired QOLIBRI scores at 12 months. This indicates that a subgroup of patients may experience physical and mental limitations one year after TBI. The pattern of HRQoL scores described in our study also indicates a ceiling effect, which is a prominent issue in TBI outcome studies [4]. 
The strongest predictors of the MCS were pre-injury mental health, level of education, and employment. Based on our findings, we can conclude that patient-related characteristics are more important for the prediction of MCS than injury-related characteristics, such as GCS. In other words, patients' wellbeing following TBI is more strongly influenced by psychosocial factors than the severity of injury. Furthermore, predictors of functional outcomes differ for patients with mild versus moderate and severe TBI, motivating the development of separate models for these patients [21]. It has been suggested that following moderate and severe TBI, functional outcome is determined by what "the injury brings to the patient", whereas in mild TBI it is determined by what "the patient brings to the injury" [23]. In contrast, predictors of HRQoL did not significantly differ between patients with mild, and moderate and severe TBI. This might be explained by the fact that HRQoL captures the patient's subjective experience of their wellbeing in daily life, and is therefore likely to be affected by psychological factors and emotional adjustment. Consequently, patientrelated characteristics (e.g., pre-injury mental health, level of education, and employment) were expected to influence HRQoL and predictor effects to vary less by injury severity.

The combined rate of pre-injury mental health problems (Anxiety, depression, sleep disorders, and schizophrenia) was $10 \%$, which is somewhat lower than pre-injury mental health problems of 19\% and 13\% for anxiety and depression based on structured diagnostic interviews) (Scholten 2016). Between studies, there is a wide variation in prevalence rates of pre-injury anxiety and depressive disorders. This can be explained by differences in study design, patients characteristics, definitions, assessment methods, and measures used to assess psychiatric outcomes.

The models for PCS performed better than those predicting MCS and the QOLIBRI total score ( $R^{2} 19 \%$ compared to $9 \%$ and $13 \%$ of the full models for MCS and QOLIBRI). Patients' resilience, coping strategies and social support are associated with psychological outcome following TBI [47-50]. Although these psychological processes are typically not assessed in RCTs or observational studies in TBI they have the potential to improve model performance and provide opportunities for focused interventions to improve long-term psychological outcome following TBI. In patients following mild TBI, post-concussion symptoms, relating to a subset of somatic, cognitive, behavioral and emotional symptoms, are negatively associated with HRQoL [51]. Furthermore, cognitive impairments are associated with HRQoL following TBI [52]. Future research should therefore focus on the development of dynamic prediction models for HRQoL following TBI, including resilience, social support, coping, cognitive impairments, and early post-concussion symptoms as (longitudinal) predictors.
The models developed in our study include characteristics that were available at admission and 2 weeks post injury. Reliable information about prognosis is of major importance to patients who sustained TBI and their families. For clinicians it would be notoriously difficult, if not impossible, to predict a patient's subjective experience of their sequelae in daily life. Prediction models for HRQoL following TBI have the potential to support clinicians to identify patients at increased risk of experiencing limitations in their daily life, who could then be followed more closely and receive early interventions to alleviate the burden of injury. Before prediction models can be considered for implementation in clinical practice, external validation is required to evaluate their performance in new settings.

Strengths of this study include the use of a longitudinal, prospective observational cohort study (CENTERTBI). Consequently, we made use of a standardized collection of data, and a well-described contemporary cohort of patients. Also, the large sample size of the development cohort allowed for reliable predictions. Another strength is the selection of candidate predictors based on literature and expert knowledge, which is preferred over selection based on data, that may increase the risk for overfitting. The predictors can be easily extracted from patients with standardized questionnaires at admission and early after admission, and are available at the time the model is to be used. Furthermore, we used a generic (SF-36v2) and TBI-specific (QOLIBRI) instrument to assess HRQoL. The SF-36 is validated and most widely used in HRQoL studies and in practice [6]. The proposed models for the SF-36v2 scales can be compared to models for other neurological conditions, such as stroke. Prior research indicates that the QOLIBRI provides additional information to the SF-36 [19].

Several limitations of our study have to be considered. First, candidate predictors were based on literature and expert knowledge. However, among studies, participants, definitions of (HR)QoL, instruments, and time points of HRQoL assessment vary widely [6]. Although prior evidence of predictors is therefore limited our study provides insight in predictors of HRQoL following TBI based on multivariable analysis. Second, living arrangement at admission was considered a proxy of social support and therefore included as a predictor. Social support is associated with psychological outcomes after TBI [50], but it is typically unmeasured in longitudinal studies. Living arrangement might be related to social support; however, we cannot generalize our findings to the effect of social support on HRQoL following TBI. Third, in our study, non-responders were more frequently diagnosed with moderate/severe TBI than responders. Patients with more severe injury might be unable to respond to questionnaires over time. Furthermore, the SF-36v2 is not suitable for patients with major cognitive impairment or language difficulties, and thus an important 
subgroup of patients with profound disability is excluded. In the future, options to further improve adherence rates among TBI patients should be explored. For instance, researchers and clinicians could combine patients' healthcare facility visits with reminders to fill in questionnaires or electronic reminders via smartphone applications.

\section{Conclusion}

Whereas prognostic models for functional outcome following TBI typically include medical and injury-related characteristics, our results suggest that patient-related characteristics contribute to the prediction of HRQoL following TBI. Prediction models for HRQoL have the potential to inform clinicians and patients and their families about prognosis 6 months after TBI. However, performance of the proposed models was moderate, which reflects the complexity of predicting HRQoL following TBI.

Supplementary Information The online version contains supplementary material available at https://doi.org/10.1007/s11136-021-02932-z.

Acknowledgements We would like to thank Daan Nieboer and Benjamin Gravesteijn for their statistical expertise that have supported the analyses performed in our study. Furthermore, we are grateful to all patients and investigators that participated in the CENTER-TBI study.

The CENTER-TBI participants and investigators: Cecilia Åkerlund (Department of Physiology and Pharmacology, Section of Perioperative Medicine and Intensive Care, Karolinska Institutet, Stockholm, Sweden: cecilia.ai.akerlund@gmail.com), Krisztina Amrein (János Szentágothai Research Centre, University of Pécs, Pécs, Hungary: tina. amrein84@gmail.com), Nada Andelic (Division of Surgery and Clinical Neuroscience, Department of Physical Medicine and Rehabilitation, Oslo University Hospital and University of Oslo, Oslo, Norway: NADAND@ous-hf.no), Lasse Andreassen (Department of Neurosurgery, University Hospital Northern Norway, Tromso, Norway: Lasse. Andreassen@unn.no), Audny Anke (Department of Physical Medicine and Rehabilitation, University Hospital Northern Norway, Tromso, Norway: Audny.anke@unn.no), Anna Antoni (Trauma Surgery, Medical University Vienna, Vienna, Austria: anna.antoni@meduniwien. ac.at), Gérard Audibert (Department of Anesthesiology \& Intensive Care, University Hospital Nancy, Nancy, France: g.audibert@chunancy.fr), Philippe Azouvi (Raymond Poincare Hospital, Assistance Publique - Hopitaux de Paris, Paris, France: philippe.azouvi@rpc. aphp.fr), Maria Luisa Azzolini (Department of Anesthesiology \& Intensive Care, S Raffaele University Hospital, Milan, Italy: azzolini. marialuisa@hsr.it), Ronald Bartels (Department of Neurosurgery, Radboud University Medical Center, Nijmegen, The Netherlands: Ronald. Bartels@radboudumc.nl), Pál Barzó (Department of Neurosurgery, University of Szeged, Szeged, Hungary: pbarzo@gmail.com), Romuald Beauvais (International Projects Management, ARTTIC, Munchen, Germany: beauvais@arttic.eu), Ronny Beer (Department of Neurology, Neurological Intensive Care Unit, Medical University of Innsbruck, Innsbruck, Austria: ronny.beer@i-med.ac.at), Bo-Michael Bellander (Department of Neurosurgery \& Anesthesia \& Intensive Care Medicine, Karolinska University Hospital, Stockholm, Sweden: bo-michael. bellander@karolinska.se), Antonio Belli (NIHR Surgical Reconstruction and Microbiology Research Centre, Birmingham, UK: a.belli@ bham.ac.uk), Habib Benali (Anesthesie-Réanimation, Assistance Publique - Hopitaux de Paris, Paris, France: habib.benali@gmail.com), Maurizio Berardino (Department of Anesthesia \& ICU, AOU Città della Salute e della Scienza di Torino - Orthopedic and Trauma Center, Torino, Italy: maurizio_berardino@fastwebnet.it), Luigi Beretta (Department of Anesthesiology \& Intensive Care, S Raffaele University Hospital, Milan, Italy: beretta.luigi@ @sr.it), Morten Blaabjerg (Department of Neurology, Odense University Hospital, Odense, Denmark: morten.blaabjerg1@ rsyd.dk), Peter Bragge (BehaviourWorks Australia, Monash Sustainability Institute, Monash University, Victoria, Australia: peter.bragge@monash.edu), Alexandra Brazinova (Department of Public Health, Faculty of Health Sciences and Social Work, Trnava University, Trnava, Slovakia: alexandra.brazinova@ gmail.com), Vibeke Brinck (Quesgen Systems Inc., Burlingame, California, USA: vibeke.brinck@ quesgen.com), Joanne Brooker (Australian \& New Zealand Intensive Care Research Centre, Department of Epidemiology and Preventive Medicine, School of Public Health and Preventive Medicine, Monash University, Melbourne, Australia: Joanne.Brooker@monash.edu), Camilla Brorsson (Department of Surgery and Perioperative Science, Umeå University, Umeå, Sweden: Camilla.Brorsson@umu.se), Andras Buki (Department of Neurosurgery, Medical School, University of Pécs, Hungary and Neurotrauma Research Group, János Szentágothai Research Centre, University of Pécs, Hungary: 2saturn@gmail.com), Monika Bullinger (Department of Medical Psychology, Universitätsklinikum Hamburg-Eppendorf, Hamburg, Germany: bullinger@uke.de), Manuel Cabeleira (Brain Physics Lab, Division of Neurosurgery, Dept of Clinical Neurosciences, University of Cambridge, Addenbrooke's Hospital, Cambridge, UK: mc916@cam.ac.uk), Alessio Caccioppola (Neuro ICU, Fondazione IRCCS Cà Granda Ospedale Maggiore Policlinico, Milan, Italy: alessio.caccioppola@gmail.com), Emiliana Calappi (Neuro ICU, Fondazione IRCCS Cà Granda Ospedale Maggiore Policlinico, Milan, Italy: calemy02@yahoo.it), Maria Rosa Calvi (Department of Anesthesiology \& Intensive Care, S Raffaele University Hospital, Milan, Italy: calvi.mariarosa@ hsr.it), Peter Cameron (ANZIC Research Centre, Monash University, Department of Epidemiology and Preventive Medicine, Melbourne, Victoria, Australia: peter.cameron@med. monash.edu.au), Guillermo Carbayo Lozano (Department of Neurosurgery, Hospital of Cruces, Bilbao, Spain: guillermobilbo@gmail. com), Marco Carbonara (Neuro ICU, Fondazione IRCCS Cà Granda Ospedale Maggiore Policlinico, Milan, Italy: marco.carbonara@gmail. com), Simona Cavallo (Department of Anesthesia \& ICU, AOU Città della Salute e della Scienza di Torino - Orthopedic and Trauma Center, Torino, Italy: cavallosimona1@gmail.com), Giorgio Chevallard (NeuroIntensive Care, Niguarda Hospital, Milan, Italy: giorgio.chevallard@ ospedaleniguarda.it), Arturo Chieregato (NeuroIntensive Care, Niguarda Hospital, Milan, Italy: arturo.chieregato@ ospedaleniguarda. it), Giuseppe Citerio (School of Medicine and Surgery, Università Milano Bicocca, Milano, Italy, NeuroIntensive Care, ASST di Monza, Monza, Italy: giuseppe.citerio@unimib.it), Hans Clusmann (Department of Neurosurgery, Medical Faculty RWTH Aachen University, Aachen, Germany: hclusmann@ukaachen.de), Mark Coburn (Department of Anesthesiology and Intensive Care Medicine, University Hospital Bonn, Bonn, Germany: mark.coburn@ukbonn.de), Jonathan Coles (Department of Anesthesia \& Neurointensive Care, Cambridge University Hospital NHS Foundation Trust, Cambridge, UK: jpc44@ wbic.cam.ac.uk), Jamie D. Cooper (School of Public Health \& PM, Monash University and The Alfred Hospital, Melbourne, Victoria, Australia: jamie.cooper@monash.edu), Marta Correia (Radiology/MRI Department, MRC Cognition and Brain Sciences Unit, Cambridge, UK: Marta.Correia@mrc-cbu.cam.ac.uk),Amra Čović (Institute of Medical Psychology and Medical Sociology, Universitätsmedizin Göttingen, Göttingen, Germany: amra.covic@med.uni-goettingen.de), Nicola Curry (Oxford University Hospitals NHS Trust, Oxford, UK: nicola.curry@ouh.nhs.uk), Endre Czeiter (Department of Neurosurgery, Medical School, University of Pécs, Hungary and Neurotrauma 
Research Group, János Szentágothai Research Centre, University of Pécs, Hungary: endre.czeiter@gmail.com), Marek Czosnyka (Brain Physics Lab, Division of Neurosurgery, Dept of Clinical Neurosciences, University of Cambridge, Addenbrooke's Hospital, Cambridge, UK: mc141@medschl.cam.ac.uk), Claire Dahyot-Fizelier Intensive Care Unit, CHU Poitiers, Potiers, France: c.dahyot-fizelier@ chu-poitiers.fr), Paul Dark (University of Manchester NIHR Biomedical Research Centre, Critical Care Directorate, Salford Royal Hospital NHS Foundation Trust, Salford, UK: paul.m.dark@ manchester.ac.uk), Helen Dawes (Movement Science Group, Faculty of Health and Life Sciences, Oxford Brookes University, Oxford, UK: hdawes@brookes. ac.uk), Véronique De Keyser (Department of Neurosurgery, Antwerp University Hospital and University of Antwerp, Edegem, Belgium: veronique.dekeyser@uza.be), Vincent Degos (Anesthesie-Réanimation, Assistance Publique - Hopitaux de Paris, Paris, France: vincent. degos@aphp.fr), Francesco Della Corte (Department of Anesthesia \& Intensive Care, Maggiore Della Carità Hospital, Novara, Italy: dellacorte.f@gmail.com), Hugo den Boogert (Department of Neurosurgery, Radboud University Medical Center, Nijmegen, The Netherlands: Hugo.denBoogert@ radboudumc.nl), Bart Depreitere (Department of Neurosurgery, University Hospitals Leuven, Leuven, Belgium: bart.depreitere@uzleuven.be), Đula Đilvesi (Department of Neurosurgery, Clinical Centre of Vojvodina, Faculty of Medicine, University of Novi Sad, Novi Sad, Serbia: djuladjilvesi@gmail.com), Abhishek Dixit (Division of Anaesthesia, University of Cambridge, Addenbrooke's Hospital, Cambridge, UK: ad825@ cam.ac.uk), Emma Donoghue (Australian \& New Zealand Intensive Care Research Centre, Department of Epidemiology and Preventive Medicine, School of Public Health and Preventive Medicine, Monash University, Melbourne, Australia: emma. donoghue@monash.edu), Jens Dreier (Center for Stroke Research Berlin, Charité - Universitätsmedizin Berlin, Corporate Member of Freie Universität Berlin, Humboldt-Universität zu Berlin, and Berlin Institute of Health, Berlin, Germany: jens.dreier@ charite.de), Guy-Loup Dulière (Intensive Care Unit, CHR Citadelle, Liège, Belgium: glduliere@gmail.com), Ari Ercole (Division of Anaesthesia, University of Cambridge, Addenbrooke's Hospital, Cambridge, UK: ae105@cam. ac.uk), Patrick Esser (Movement Science Group, Faculty of Health and Life Sciences, Oxford Brookes University, Oxford, UK: pesser@ brookes.ac.uk), Erzsébet Ezer (Department of Anaesthesiology and Intensive Therapy, University of Pécs, Pécs, Hungary: ezererzsebet@ yahoo.com), Martin Fabricius (Departments of Neurology, Clinical Neurophysiology and Neuroanesthesiology, Region Hovedstaden Rigshospitalet, Copenhagen, Denmark: fabricius@dadlnet.dk), Valery L. Feigin (National Institute for Stroke and Applied Neurosciences, Faculty of Health and Environmental Studies, Auckland University of Technology, Auckland, New Zealand: valery.feigin@aut.ac.nz), Kelly Foks (Department of Neurology, Erasmus MC, Rotterdam, The Netherlands: k.foks@erasmusmc.nl), Shirin Frisvold (Department of Anesthesiology and Intensive care, University Hospital Northern Norway, Tromso, Norway: Shirin.Kordasti@unn.no), Alex Furmanov (Department of Neurosurgery, Hadassah-Hebrew University Medical Center, Jerusalem, Israel: alexpuil@yahoo.com), Pablo Gagliardo (Fundación Instituto Valenciano de Neurorrehabilitación (FIVAN), Valencia, Spain: pablog@ fivan.org), Damien Galanaud(Anesthesie-Réanimation, Assistance Publique - Hopitaux de Paris, Paris, France: galanaud@ gmail.com), Dashiell Gantner (ANZIC Research Centre, Monash University, Department of Epidemiology and Preventive Medicine, Melbourne, Victoria, Australia: dashiell.gantner@monash.edu), Guoyi Gao (Department of Neurosurgery, Shanghai Renji Hospital, Shanghai Jiaotong University/School of Medicine, Shanghai, China: gao3@sina. com), Pradeep George (Karolinska Institutet, INCF International Neuroinformatics Coordinating Facility, Stockholm, Sweden: george@ incf. org), Alexandre Ghuysen (Emergency Department, CHU, Liège, Belgium: A.Ghuysen@chu.ulg.ac.be), Lelde Giga (Neurosurgery Clinic, Pauls Stradins Clinical University Hospital, Riga, Latvia: lelde.giga@ inbox.lv), Ben Glocker (Department of Computing, Imperial College
London, London, UK: b.glocker@imperial.ac.uk), Jagoš Golubovic (Department of Neurosurgery, Clinical Centre of Vojvodina, Faculty of Medicine, University of Novi Sad, Novi Sad, Serbia: jagosgolubovic@ gmail.com), Pedro A. Gomez (Department of Neurosurgery, Hospital Universitario 12 de Octubre, Madrid, Spain: A.pagolopez@ gmail.com), Johannes Gratz (Department of Anesthesia, Critical Care and Pain Medicine, Medical University of Vienna, Austria: johannes. gratz@meduniwien.ac.at), Benjamin Gravesteijn (Department of Public Health, Erasmus Medical Center-University Medical Center, Rotterdam, The Netherlands: b.gravesteijn@erasmusmc.nl), Francesca Grossi (Department of Anesthesia \& Intensive Care, Maggiore Della Carità Hospital, Novara, Italy: francesca.grossi@ libero.it), Russell L. Gruen (College of Health and Medicine, Australian National University, Canberra, Australia: russell.gruen@anu.edu.au), Deepak Gupta (Department of Neurosurgery, Neurosciences Centre \& JPN Apex Trauma Centre, All India Institute of Medical Sciences, New Delhi110029, India: drdeepakgupta@gmail.com), Juanita A. Haagsma (Department of Public Health, Erasmus Medical Center-University Medical Center, Rotterdam, The Netherlands: j.haagsma@erasmusmc. nl), Iain Haitsma (Department of Neurosurgery, Erasmus MC, Rotterdam, The Netherlands: i.haitsma@erasmusmc.nl), Raimund Helbok (Department of Neurology, Neurological Intensive Care Unit, Medical University of Innsbruck, Innsbruck, Austria: Raimund.Helbok@tirolkliniken.at), Eirik Helseth (Department of Neurosurgery, Oslo University Hospital, Oslo, Norway: EHELSETH@ ous-hf.no), Lindsay Horton (Division of Psychology, University of Stirling, Stirling, UK: lindsay. horton@stir.ac.uk), Jilske Huijben (Department of Public Health, Erasmus Medical Center-University Medical Center, Rotterdam, The Netherlands: j.a.huijben@erasmusmc.nl), Peter J. Hutchinson (Division of Neurosurgery, Department of Clinical Neurosciences, Addenbrooke's Hospital \& University of Cambridge, Cambridge, UK: pjah2@cam. ac.uk), Bram Jacobs (Department of Neurology, University of Groningen, University Medical Center Groningen, Groningen, Netherlands: b.jacobs@umcg.nl), Stefan Jankowski (Neurointensive Care, Sheffield Teaching Hospitals NHS Foundation Trust, Sheffield, UK: Stefan. Jankowski@sth.nhs.uk), Mike Jarrett (Quesgen Systems Inc., Burlingame, California, USA: mike.jarrett@quesgen.com), Ji-yao Jiang (Karolinska Institutet, INCF International Neuroinformatics Coordinating Facility, Stockholm, Sweden: jiyaojiang@ 126.com), Faye Johnson (Salford Royal Hospital NHS Foundation Trust Acute Research Delivery Team, Salford, UK: faye.johnson@live.co.uk), Kelly Jones (National Institute for Stroke and Applied Neurosciences, Faculty of Health and Environmental Studies, Auckland University of Technology, Auckland, New Zealand: kejones@aut.ac.nz), Mladen Karan (Department of Neurosurgery, Clinical Centre of Vojvodina, Faculty of Medicine, University of Novi Sad, Novi Sad, Serbia: mladjokaran@ gmail.com), Angelos G. Kolias (Division of Neurosurgery, Department of Clinical Neurosciences, Addenbrooke's Hospital \& University of Cambridge, Cambridge, UK: angeloskolias@ gmail.com), Erwin Kompanje (Department of Intensive Care and Department of Ethics and Philosophy of Medicine, Erasmus Medical Center, Rotterdam, The Netherlands: erwinkompanje@me.com), Daniel Kondziella (Departments of Neurology, Clinical Neurophysiology and Neuroanesthesiology, Region Hovedstaden Rigshospitalet, Copenhagen, Denmark: Daniel.Kondziella@ regionh.dk), Evgenios Kornaropoulos (Division of Anaesthesia, University of Cambridge, Addenbrooke's Hospital, Cambridge, UK: ek481@cam.ac.uk), Lars-Owe Koskinen (Department of Clinical Neuroscience, Neurosurgery, Umeå University, Umeå, Sweden: Lars-Owe.Koskinen@umu.se), Noémi Kovács (Hungarian Brain Research Program - Grant No. KTIA_13_NAP-A-II/8, University of Pécs, Pécs, Hungary: kovacs.noemi@pte.hu), Ana Kowark (Department of Anaesthesiology, University Hospital of Aachen, Aachen, Germany: akowark@ukaachen.de), Alfonso Lagares (Department of Neurosurgery, Hospital Universitario 12 de Octubre, Madrid, Spain: algadoc@yahoo.com), Linda Lanyon (Karolinska Institutet, INCF International Neuroinformatics Coordinating Facility, Stockholm, 
Sweden: lindal@incf.org), Steven Laureys (Cyclotron Research Center, University of Liège, Liège, Belgium: steven.laureys@ulg.ac.be), Fiona Lecky (Centre for Urgent and Emergency Care Research (CURE), Health Services Research Section, School of Health and Related Research (ScHARR), University of Sheffield, Sheffield, UK, Emergency Department, Salford Royal Hospital, Salford, UK: f.e.lecky@ sheffield.ac.uk), Didier Ledoux (Cyclotron Research Center, University of Liège, Liège, Belgium: dledoux @ chu.ulg.ac.be), Rolf Lefering (Institute of Research in Operative Medicine (IFOM), Witten/Herdecke University, Cologne, Germany: Rolf.Lefering@uni-wh.de), Valerie Legrand (VP Global Project Management CNS, ICON, Paris, France: Valerie.Legrand@iconplc.com), Aurelie Lejeune (Department of Anesthesiology-Intensive Care, Lille University Hospital, Lille, France: aurelie.lejeune@chru-lille.fr), Leon Levi (Department of Neurosurgery, Rambam Medical Center, Haifa, Israel: 1levi@ rambam.health. gov.il), Roger Lightfoot (Department of Anesthesiology \& Intensive Care, University Hospitals Southampton NHS Trust, Southampton, UK: Roger.Lightfoot@uhs.nhs.uk), Hester Lingsma (Department of Public Health, Erasmus Medical Center-University Medical Center, Rotterdam, The Netherlands: h.lingsma@erasmusmc.nl), Andrew I.R. Maas (Department of Neurosurgery, Antwerp University Hospital and University of Antwerp, Edegem, Belgium: I.R. andrew.maas@uza.be), Ana M. Castaño-León (Department of Neurosurgery, Hospital Universitario 12 de Octubre, Madrid, Spain: ana.maria.castano.leon@gmail. com), Marc Maegele (Cologne-Merheim Medical Center (CMMC), Department of Traumatology, Orthopedic Surgery and Sportmedicine, Witten/Herdecke University, Cologne, Germany: Marc.Maegele@tonline.de), Marek Majdan (Department of Public Health, Faculty of Health Sciences and Social Work, Trnava University, Trnava, Slovakia: mmajdan@truni.sk), Alex Manara (Intensive Care Unit, Southmead Hospital, Bristol, Bristol, UK: Alex.Manara@nbt.nhs.uk), Geoffrey Manley (Department of Neurological Surgery, University of California, San Francisco, California, USA: ManleyG@ucsf.edu), Costanza Martino (Department of Anesthesia \& Intensive Care, M. Bufalini Hospital, Cesena, Italy: costmartino74@gmail.com), Hugues Maréchal (Intensive Care Unit, CHR Citadelle, Liège, Belgium: Hugues.Marechal@ chrcitadelle.be), Julia Mattern (Department of Neurosurgery, University Hospital Heidelberg, Heidelberg, Germany: Julia.Mattern@ med. uni-heidelberg.de), Catherine McMahon (Department of Neurosurgery, The Walton Centre NHS Foundation Trust, Liverpool, UK: Catherine. McMahon@thewaltoncentre.nhs.uk), Béla Melegh (Department of Medical Genetics, University of Pécs, Pécs, Hungary: bela.melegh@ aok.pte.hu), David Menon (Division of Anaesthesia, University of Cambridge, Addenbrooke's Hospital, Cambridge, UK: dkm13@cam. ac.uk), Tomas Menovsky (Department of Neurosurgery, Antwerp University Hospital and University of Antwerp, Edegem, Belgium: tomas. menovsky@uza.be), Ana Mikolic (Department of Public Health, Erasmus Medical Center-University Medical Center, Rotterdam, The Netherlands: a.mikolic@erasmusmc.nl),Benoit Misset (Cyclotron Research Center, University of Liège, Liège, Belgium: Benoit.Misset@ chuliege. be), Visakh Muraleedharan (Karolinska Institutet, INCF International Neuroinformatics Coordinating Facility, Stockholm, Sweden: visakh@ incf.org), Lynnette Murray (ANZIC Research Centre, Monash University, Department of Epidemiology and Preventive Medicine, Melbourne, Victoria, Australia: lynnette.murray@monash.edu), Ancuta Negru (Department of Neurosurgery, Emergency County Hospital Timisoara, Timisoara, Romania: negruancu@gmail.com), David Nelson (Department of Physiology and Pharmacology, Section of Perioperative Medicine and Intensive Care, Karolinska Institutet, Stockholm, Sweden: david.nelson@karolinska.se), Virginia Newcombe (Division of Anaesthesia, University of Cambridge, Addenbrooke's Hospital, Cambridge, UK: vfin2@cam.ac.uk), Daan Nieboer (Department of Public Health, Erasmus Medical Center-University Medical Center, Rotterdam, The Netherlands: d.nieboer@erasmusmc.nl), József Nyirádi (János Szentágothai Research Centre, University of Pécs, Pécs, Hungary: nyiradi.jozsef@pte.hu), Otesile Olubukola (Centre for Urgent and
Emergency Care Research (CURE), Health Services Research Section, School of Health and Related Research (ScHARR), University of Sheffield, Sheffield, UK: o.otesile@ sheffield.ac.uk), Matej Oresic (School of Medical Sciences, Örebro University, Örebro, Sweden: matej. oresic@oru.se), Fabrizio Ortolano (Neuro ICU, Fondazione IRCCS Cà Granda Ospedale Maggiore Policlinico, Milan, Italy: lupeda@gmail. com), Aarno Palotie (Institute for Molecular Medicine Finland, University of Helsinki, Helsinki, Finland, Analytic and Translational Genetics Unit, Department of Medicine; Psychiatric \& Neurodevelopmental Genetics Unit, Department of Psychiatry; Department of Neurology, Massachusetts General Hospital, Boston, MA, USA, Program in Medical and Population Genetics; The Stanley Center for PsychiatricResearch, The Broad Institute of MIT and Harvard, Cambridge, MA, USA: aarno.palotie@ helsinki.fi), Paul M. Parizel (Department of Radiology, University of Antwerp, Edegem, Belgium: paul.parizel@ uantwerpen.be), Jean-François Payen (Department of Anesthesiology \& Intensive Care, University Hospital of Grenoble, Grenoble, France: Jean-Francois.Payen@ujf-grenoble.fr), Natascha Perera (International Projects Management, ARTTIC, Munchen, Germany: perera@arttic. eu), Vincent Perlbarg (Anesthesie-Réanimation, Assistance Publique - Hopitaux de Paris, Paris, France: vincent.perlbarg@gmail.com), Paolo Persona (Department of Anesthesia \& Intensive Care, Azienda Ospedaliera Università di Padova, Padova, Italy: ppersona75@gmail. com), Wilco Peul (Dept. of Neurosurgery, Leiden University Medical Center, Leiden, The Netherlands and Dept. of Neurosurgery, Medical Center Haaglanden, The Hague, The Netherlands: W.C.Peul@lumc. nl), Anna Piippo-Karjalainen (Department of Neurosurgery, Helsinki University Central Hospital: Anna anna.piippo@ @us.fi), Matti Pirinen (Institute for Molecular Medicine Finland, University of Helsinki, Helsinki, Finland: matti.pirinen@ helsinki.fi), Dana Pisica (Department of Public Health, Erasmus Medical Center-University Medical Center, Rotterdam, The Netherlands: d.pisica@erasmusmc.nl), Horia Ples (Department of Neurosurgery, Emergency County Hospital Timisoara, Timisoara, Romania: horia.ples@ neuromed.ro), Suzanne Polinder (Department of Public Health, Erasmus Medical Center-University Medical Center, Rotterdam, The Netherlands: s.polinder@erasmusmc. nl), Inigo Pomposo (Department of Neurosurgery, Hospital of Cruces, Bilbao, Spain: inigo.pomposo@osakidetza.net), Jussi P. Posti (Division of Clinical Neurosciences, Department of Neurosurgery and Turku Brain Injury Centre, Turku University Hospital and University of Turku, Turku, Finland: jussi.posti@ tyks.fi), Louis Puybasset (Department of Anesthesiology and Critical Care, Pitié -Salpêtrière Teaching Hospital, Assistance Publique, Hôpitaux de Paris and University Pierre et Marie Curie, Paris, France: louis.puybasset@aphp.fr), Andreea Radoi (Neurotraumatology and Neurosurgery Research Unit (UNINN), Vall d'Hebron Research Institute, Barcelona, Spain: aradoi@neurotrauma.net), Arminas Ragauskas (Department of Neurosurgery, Kaunas University of Technology and Vilnius University, Vilnius, Lithuania: telematics@ktu.lt), Rahul Raj (Department of Neurosurgery, Helsinki University Central Hospital: rahul.raj@hus.fi), Malinka Rambadagalla (Department of Neurosurgery, Rezekne Hospital, Latvia: malinka.rambadagalla@gmail.com), Isabel Retel Helmrich (Department of Public Health, Erasmus Medical Center-University Medical Center, Rotterdam, The Netherlands: i.retelhelmrich@erasmusmc.nl), Jonathan Rhodes (Department of Anaesthesia, Critical Care \& Pain Medicine NHS Lothian \& University of Edinburg, Edinburgh, UK: jrhodes $1 @$ staffmail.ed.ac.uk), Sylvia Richardson (Director, MRC Biostatistics Unit, Cambridge Institute of Public Health, Cambridge, UK: sylvia.richardson@mrc-bsu.cam.ac.uk), Sophie Richter (Division of Anaesthesia, University of Cambridge, Addenbrooke's Hospital, Cambridge, UK: sr773@cam.ac.uk), Samuli Ripatti (Institute for Molecular Medicine Finland, University of Helsinki, Helsinki, Finland: samuli.ripatti@ helsinki.fi), Saulius Rocka (Department of Neurosurgery, Kaunas University of Technology and Vilnius University, Vilnius, Lithuania: saulius.rocka@mf.vu.lt), Cecilie Roe (Department of Physical Medicine and Rehabilitation, Oslo University Hospital/University of Oslo, Oslo, 
Norway: e.c.t.roe@medisin.uio.no), Olav Roise (Division of Orthopedics, Oslo University Hospital, Oslo, Norway, Institute of Clinical Medicine, Faculty of Medicine, University of Oslo, Oslo, Norway: olav. roise@medisin.uio.no), Jonathan Rosand (Broad Institute, Cambridge MA Harvard Medical School, Boston MA, Massachusetts General Hospital, Boston, MA, USA: jrosand @ partners.org), Jeffrey V. Rosenfeld (National Trauma Research Institute, The Alfred Hospital, Monash University, Melbourne, Victoria, Australia: J.Rosenfeld@ alfred.org. au), Christina Rosenlund (Department of Neurosurgery, Odense University Hospital, Odense, Denmark: chrisstenrose@gmail.com), Guy Rosenthal (Department of Neurosurgery, Hadassah-Hebrew University Medical Center, Jerusalem, Israel: rosenthalg@ hadassah.org.il), Rolf Rossaint (Cyclotron Research Center, University of Liège, Liège, Belgium: RRossaint@ukaachen.de), Sandra Rossi (Department of Anesthesia \& Intensive Care, Azienda Ospedaliera Università di Padova, Padova, Italy: sandrarossi0@ gmail.com), Daniel Rueckert (Department of Computing, Imperial College London, London, UK: d.rueckert@ imperial.ac.uk) Martin Rusnák (International Neurotrauma Research Organisation, Vienna, Austria: mrusnak@igeh.org), Juan Sahuquillo (Neurotraumatology and Neurosurgery Research Unit (UNINN), Vall d'Hebron Research Institute, Barcelona, Spain: sahuquillo@ neurotrauma.net), Oliver Sakowitz (Department of Neurosurgery, University Hospital Heidelberg, Heidelberg, Germany, Klinik für Neurochirurgie, Klinikum Ludwigsburg, Ludwigsburg, Germany: oliver.sakowitz@ gmail.com), Renan Sanchez-Porras (Klinik für Neurochirurgie, Klinikum Ludwigsburg, Ludwigsburg, Germany: renan_md@hotmail.com), Janos Sandor (Division of Biostatistics and Epidemiology, Department of Preventive Medicine, University of Debrecen, Debrecen, Hungary: sandor.janos@sph.unideb.hu), Nadine Schäfer (Institute of Research in Operative Medicine (IFOM), Witten/Herdecke University, Cologne, Germany: Nadine.Schaefer@uni-wh.de), Silke Schmidt (Department Health and Prevention, University Greifswald, Greifswald, Germany: silke.schmidt@uni-greifswald.de), Herbert Schoechl (Department of Anaesthesiology and Intensive Care, AUVA Trauma Hospital, Salzburg, Austria: Herbert.Schoechl@auva.at), Guus Schoonman (Department of Neurology, Elisabeth-TweeSteden Ziekenhuis, Tilburg, The Netherlands: g.schoonman@tsz.nl g.schoonman@tsz.nl), Rico Frederik Schou (Department of Neuroanesthesia and Neurointensive Care, Odense University Hospital, Odense, Denmark: rico@mymedic.dk), Elisabeth Schwendenwein (Trauma Surgery, Medical University Vienna, Vienna, Austria: elisabeth.schwendenwein@meduniwien. ac.at), Charlie Sewalt (Department of Public Health, Erasmus Medical Center-University Medical Center, Rotterdam, The Netherlands: c.sewalt@erasmusmc.nl), Toril Skandsen (Department of Neuromedicine and Movement Science, Norwegian University of Science and Technology, NTNU, Trondheim, Norway, Department of Physical Medicine and Rehabilitation, St. Olavs Hospital, Trondheim University Hospital, Trondheim, Norway: toril.skandsen@ntnu.no), Peter Smielewski (Brain Physics Lab, Division of Neurosurgery, Dept of Clinical Neurosciences, University of Cambridge, Addenbrooke's Hospital, Cambridge, UK: ps10011@cam.ac.uk), Abayomi Sorinola (Department of Neurosurgery, University of Pécs, Pécs, Hungary: sorinola_abayomi@hotmail.com), Emmanuel Stamatakis (Division of Anaesthesia, University of Cambridge, Addenbrooke's Hospital, Cambridge, UK: eas46@ cam.ac.uk), Simon Stanworth (Oxford University Hospitals NHS Trust, Oxford, UK: simon.stanworth@nhsbt.nhs.uk), Robert Stevens (Division of Neuroscience Critical Care, John Hopkins University School of Medicine, Baltimore, USA: rstevens@jhmi.edu), William Stewart (Department of Neuropathology, Queen Elizabeth University Hospital and University of Glasgow, Glasgow, UK: william. stewart@glasgow.ac.uk), Ewout W. Steyerberg (Department of Public Health, Erasmus Medical Center-University Medical Center, Rotterdam, The Netherlands, Dept. of Department of Biomedical Data Sciences, Leiden University Medical Center, Leiden, The Netherlands: e.steyerberg@erasmusmc.nl), Nino Stocchetti (Department of Pathophysiology and Transplantation, Milan University, and Neuroscience
ICU, Fondazione IRCCS Cà Granda Ospedale Maggiore Policlinico, Milano, Italy: stocchet@policlinico.mi.it), Nina Sundström (Department of Radiation Sciences, Biomedical Engineering, Umeå University, Umeå, Sweden: Nina.Sundstrom@ @ll.se), Riikka Takala (Perioperative Services, Intensive Care Medicine and Pain Management, Turku University Hospital and University of Turku, Turku, Finland: riikka.takala@tyks.fi), Viktória Tamás (Department of Neurosurgery, University of Pécs, Pécs, Hungary: tamas.viktoria@pte.hu), Tomas Tamosuitis (Department of Neurosurgery, Kaunas University of Health Sciences, Kaunas, Lithuania: tomas.tamosuitis@kaunoklinikos.lt), Mark Steven Taylor (Department of Public Health, Faculty of Health Sciences and Social Work, Trnava University, Trnava, Slovakia: marktrnava@gmail.com), Braden Te Ao (National Institute for Stroke and Applied Neurosciences, Faculty of Health and Environmental Studies, Auckland University of Technology, Auckland, New Zealand: braden. teao@aut.ac.nz), Olli Tenovuo (Division of Clinical Neurosciences, Department of Neurosurgery and Turku Brain Injury Centre, Turku University Hospital and University of Turku, Turku, Finland: olli.tenovuo@tyks.fi), Alice Theadom (National Institute for Stroke and Applied Neurosciences, Faculty of Health and Environmental Studies, Auckland University of Technology, Auckland, New Zealand: alice. theadom@aut.ac.nz), Matt Thomas (Intensive Care Unit, Southmead Hospital, Bristol, Bristol, UK: Matt.Thomas@nbt.nhs.uk), Dick Tibboel (Intensive Care and Department of Pediatric Surgery, Erasmus Medical Center, Sophia Children's Hospital, Rotterdam, The Netherlands: d.tibboel@erasmusmc.nl), Marjolein Timmers (Department of Intensive Care and Department of Ethics and Philosophy of Medicine, Erasmus Medical Center, Rotterdam, The Netherlands: mtimmers@ hotmail.com), Christos Tolias (Department of Neurosurgery, Kings College London, London, UK: christos.tolias@nhs.net), Tony Trapani (ANZIC Research Centre, Monash University, Department of Epidemiology and Preventive Medicine, Melbourne, Victoria, Australia: tony.trapani@monash.edu), Cristina Maria Tudora (Department of Neurosurgery, Emergency County Hospital Timisoara, Timisoara, Romania: cristina.tudora@ neuromed.ro), Andreas Unterberg (Department of Neurosurgery, University HospitalHeidelberg, Heidelberg, Germany: Andreas.Unterberg@med.uni-heidelberg.de), Peter Vajkoczy (Neurologie, Neurochirurgie und Psychiatrie, Charité - Universitätsmedizin Berlin, Berlin, Germany: Peter.Vajkoczy@charite.de), Shirley Vallance (ANZIC Research Centre, Monash University, Department of Epidemiology and Preventive Medicine, Melbourne, Victoria, Australia: S.Vallance@alfred.org.au), Egils Valeinis (Neurosurgery Clinic, Pauls Stradins Clinical University Hospital, Riga, Latvia: Egils. Valeinis@latnet.lv), Zoltán Vámos (Department of Anaesthesiology and Intensive Therapy, University of Pécs, Pécs, Hungary: azozoka@ gmail.com), Mathieu van der Jagt (Department of Intensive Care Adults, Erasmus MC- University Medical Center Rotterdam, Rotterdam, The Netherlands: m.vanderjagt@erasmusmc.nl), Gregory Van der Steen (Department of Neurosurgery, Antwerp University Hospital and University of Antwerp, Edegem, Belgium: gregory@webstone.be), Joukje van der Naalt (Department of Neurology, University of Groningen, University Medical Center Groningen, Groningen, Netherlands: j.van.der.naalt@umcg.nl), Jeroen T.J.M. van Dijck (Dept. of Neurosurgery, Leiden University Medical Center, Leiden, The Netherlands and Dept. of Neurosurgery, Medical Center Haaglanden, The Hague, The Netherlands: j.van.dijck@ haaglandenmc.nl), Thomas A. van Essen (Dept. of Neurosurgery, Leiden University Medical Center, Leiden, The Netherlands and Dept. of Neurosurgery, Medical Center Haaglanden, The Hague, The Netherlands: T.A.van_Essen@lumc.nl), Wim Van Hecke (icoMetrix NV, Leuven, Belgium: wim.vanhecke@icometrix. com), Caroline van Heugten (Movement Science Group, Faculty of Health and Life Sciences, Oxford Brookes University, Oxford, UK: Caroline.vanheugten@maastrichtuniversity.nl), Dominique Van Praag (Psychology Department, Antwerp University Hospital, Edegem, Belgium: Dominique dominique.vanpraag@uza.be), Ernest van Veen (Department of Public Health, Erasmus Medical Center-University 
Medical Center, Rotterdam, The Netherlands: e.vanveen.1@erasmusmc.nl), Thijs Vande Vyvere (icoMetrix NV, Leuven, Belgium: thijs. vandevyvere@icometrix.com), Roel P. J. van Wijk (Dept. of Neurosurgery, Leiden University Medical Center, Leiden, The Netherlands and Dept. of Neurosurgery, Medical Center Haaglanden, The Hague, The Netherlands: roel-van-wijk@ziggo.nl), Alessia Vargiolu (NeuroIntensive Care, ASST di Monza, Monza, Italy: neurorianimazione@ hsgerardo.org), Emmanuel Vega (Department of Anesthesiology-Intensive Care, Lille University Hospital, Lille, France: emmanuel.vega@ chru-lille.fr), Kimberley Velt (Department of Public Health, Erasmus Medical Center-University Medical Center, Rotterdam, The Netherlands: k.velt@erasmusmc.nl), Jan Verheyden (icoMetrix NV, Leuven, Belgium: jan.verheyden@icometrix.com), Paul M. Vespa (Director of Neurocritical Care, University of California, Los Angeles, USA: PVespa@mednet.ucla.edu), Anne Vik (Department of Neuromedicine and Movement Science, Norwegian University of Science and Technology, NTNU, Trondheim, Norway, Department of Neurosurgery, St. Olavs Hospital, Trondheim University Hospital, Trondheim, Norway: anne.vik@ntnu.no), Rimantas Vilcinis (Department of Neurosurgery, Kaunas University of Health Sciences, Kaunas, Lithuania: rimantas. vilcinis@kaunoklinikos.lt), Victor Volovici (Department of Neurosurgery, Erasmus MC, Rotterdam, The Netherlands: v.volovici@erasmusmc.nl), Nicole von Steinbüchel (Institute of Medical Psychology and Medical Sociology, Universitätsmedizin Göttingen, Göttingen, Germany: nvsteinbuechel@ med.uni-goettingen.de), Daphne Voormolen (Department of Public Health, Erasmus Medical Center-University Medical Center, Rotterdam, The Netherlands: d.voormolen@erasmusmc.nl), Petar Vulekovic (Department of Neurosurgery, Clinical Centre of Vojvodina, Faculty of Medicine, University of Novi Sad, Novi Sad, Serbia: pvulekovic@gmail.com), Kevin K.W. Wang (Department of Emergency Medicine, University of Florida, Gainesville, Florida, USA: kawangwang17@gmail.com), Eveline Wiegers (Department of Public Health, Erasmus Medical Center-University Medical Center, Rotterdam, The Netherlands: e.wiegers@erasmusmc.nl), Guy Williams (Division of Anaesthesia, University of Cambridge, Addenbrooke's Hospital, Cambridge, UK: gbw1000@wbic.cam.ac.uk), Lindsay Wilson (Division of Psychology, University of Stirling, Stirling, UK: 1.wilson@stir.ac.uk), Stefan Winzeck (Division of Anaesthesia, University of Cambridge, Addenbrooke's Hospital, Cambridge, UK: sw742@cam.ac.uk), Stefan Wolf (Department of Neurosurgery, Charité - Universitätsmedizin Berlin, Corporate Member of Freie Universität Berlin, Humboldt-Universität zu Berlin, and Berlin Institute of Health, Berlin, Germany: stefan.wolf@ charite.de), Zhihui Yang (Broad Institute, Cambridge MA Harvard Medical School, Boston MA, Massachusetts General Hospital, Boston, MA, USA: zhihuiyang@ufl.edu), Peter Ylén (VTT Technical Research Centre, Tampere, Finland: peter. ylen@vtt.fi), Alexander Younsi (Department of Neurosurgery, University Hospital Heidelberg, Heidelberg, Germany: alexander.younsi@ med.uni-heidelberg.de), Frederick A. Zeiler (Division of Anaesthesia, University of Cambridge, Addenbrooke's Hospital, Cambridge, UK, Section of Neurosurgery, Department of Surgery, Rady Faculty of Health Sciences, University of Manitoba, Winnipeg, MB, Canada: umzeiler@myumanitoba.ca), Veronika Zelinkova (Department of Public Health, Faculty of Health Sciences and Social Work, Trnava University, Trnava, Slovakia), Agate Ziverte (Neurosurgery Clinic, Pauls Stradins Clinical University Hospital, Riga, Latvia: agate.ziverte@ inbox.lv), Tommaso Zoerle (Neuro ICU, Fondazione IRCCS Cà Granda Ospedale Maggiore Policlinico, Milan, Italy: tommaso.zoerle@ policlinico.mi.it).

Funding The research leading to these results was supported by the European Union's Seventh Framework Programme (FP7/2007-2013) under Grant Agreement n ${ }^{\circ} 602150$ (CENTER-TBI). Additional funding was obtained from the Hannelore Kohl Stiftung (Germany), from OneMind (USA), and from Integra LifeSciences Corporation (USA).

Data availability CENTER-TBI is committed to data sharing and in particular to responsible further use of the data. Hereto, we have a data sharing statement in place: https://www.center-tbi.eu/data/shari ng. The CENTER-TBI dataset is hugely complex, and the CENTER researchers wish to encourage correct and appropriate use of the data; this means that we encourage researchers to contact the CENTER-TBI Team for any research plans and the Data Curation Team for any help in appropriate use of the data, including sharing of scripts. Requests for data access can be submitted online: https://www.center-tbi.eu/data. The complete Manual for data access is also available online: https:// www.center-tbi.eu/files/SOP-Manual-DAPR-2402020.pdf.

Code availability The variable names and associated data dictionary elements for predictors and outcomes included in our study can be found here: https://center-tbi.incf.org/_60ae5959e840f1032773d145 and variable names and associated data dictionary elements for imaging data can be found here: https://center-tbi.incf.org/_60ae5a61e840f10 $32773 \mathrm{~d} 146$. $R$ code is available upon request.

\section{Declarations}

Conflict of interest The authors declare that there is no conflict of interest.

Ethical approval The CENTER-TBI study (EC Grant 602150) has been conducted in accordance with all relevant laws of the EU if directly applicable or of direct effect and all relevant laws of the country where the Recruiting sites were located, including but not limited to, the relevant privacy and data protection laws and regulations (the "Privacy Law"), the relevant laws and regulations on the use of human materials, and all relevant guidance relating to clinical studies from time to time in force including, but not limited to, the ICH Harmonised Tripartite Guideline for Good Clinical Practice (CPMP/ICH/135/95) ("ICH GCP") and the World Medical Association Declaration of Helsinki entitled "Ethical Principles for Medical Research Involving Human Subjects".

Informed consent Informed Consent by the patients and/or the legal representative/next of kin was obtained, accordingly to the local legislations, for all patients recruited in the Core Dataset of CENTER-TBI and documented in the e-CRF. Ethical approval was obtained for each recruiting site. The list of sites, Ethical Committees, approval numbers, and approval dates can be found on the website: https://www.center-tbi. eu/project/ethical-approval.

Open Access This article is licensed under a Creative Commons Attribution 4.0 International License, which permits use, sharing, adaptation, distribution and reproduction in any medium or format, as long as you give appropriate credit to the original author(s) and the source, provide a link to the Creative Commons licence, and indicate if changes were made. The images or other third party material in this article are included in the article's Creative Commons licence, unless indicated otherwise in a credit line to the material. If material is not included in the article's Creative Commons licence and your intended use is not permitted by statutory regulation or exceeds the permitted use, you will need to obtain permission directly from the copyright holder. To view a copy of this licence, visit http://creativecommons.org/licenses/by/4.0/. 


\section{References}

1. Rosenfeld, J. V., Maas, A. I., Bragge, P., Morganti-Kossmann, M. C., Manley, G. T., \& Gruen, R. L. (2012). Early management of severe traumatic brain injury. Lancet, 9847, 1088-1098.

2. Perry, D. C., Sturm, V. E., Peterson, M. J., Pieper, C. F., Bullock, T., Boeve, B. F., Miller, B. L., Guskiewicz, K. M., Berger, M. S., \& Kramer, J. H. (2016). Association of traumatic brain injury with subsequent neurological and psychiatric disease: A meta-analysis. Journal of Neurosurgery, 2, 511-526.

3. de Koning, M. E., Scheenen, M. E., van der Horn, H. J., Hageman, G., Roks, G., Spikman, J. M., \& van der Naalt, J. (2017). Non-hospitalized patients with mild traumatic brain injury: The forgotten minority. Journal of Neurotrauma, 1, 257-261.

4. Shukla, D., Devi, B. I., \& Agrawal, A. (2011). Outcome measures for traumatic brain injury. Clinical Neurology and Neurosurgery, $6,435-441$.

5. Nichol, A. D., Higgins, A. M., Gabbe, B. J., Murray, L. J., Cooper, D. J., \& Cameron, P. A. (2011). Measuring functional and quality of life outcomes following major head injury: Common scales and checklists. Injury, 3, 281-287.

6. Polinder, S., Haagsma, J. A., van Klaveren, D., Steyerberg, E. W., \& Van Beeck, E. F. (2015). Health-related quality of life after TBI: A systematic review of study design, instruments, measurement properties, and outcome. Population Health Metrics, 1, 4.

7. Dikmen, S. S., Machamer, J. E., Powell, J. M., \& Temkin, N. R. (2003). Outcome 3 to 5 years after moderate to severe traumatic brain injury. Archives of Physical Medicine and Rehabilitation, 10, 1449-1457.

8. Kozlowski, O., Pollez, B., Thevenon, A., Dhellemmes, P., \& Rousseaux, M. (2002). Outcome and quality of life after three years in a cohort of patients with severe traumatic brain injury. Annales de readaptation et de medecine physique: Revue scientifique de la Societe francaise de reeducation fonctionnelle de readaptation et de medecine physique, 45(8), 466-473.

9. Svendsen, H. A., \& Teasdale, T. W. (2006). The influence of neuropsychological rehabilitation on symptomatology and quality of life following brain injury: A controlled long-term follow-up. Brain Injury, 12, 1295-1306.

10. Wood, R. L., \& Rutterford, N. A. (2006). Psychosocial adjustment 17 years after severe brain injury. Journal of Neurology, Neurosurgery and Psychiatry, 1, 71-73.

11. Scholten, A. C., Haagsma, J. A., Andriessen, T., Vos, P. E., Steyerberg, E. W., Van Beeck, E. F., \& Polinder, S. (2015). Healthrelated quality of life after mild, moderate and severe traumatic brain injury: Patterns and predictors of suboptimal functioning during the first year after injury. Injury, 4, 616-624.

12. Teasdale, T. W., \& Engberg, A. W. (2005). Subjective well-being and quality of life following traumatic brain injury in adults: A long-term population-based follow-up. Brain Injury, 12, 1041-1048.

13. Andelic, N., Hammergren, N., Bautz-Holter, E., Sveen, U., Brunborg, C., \& Røe, C. (2009). Functional outcome and health-related quality of life 10 years after moderate-to-severe traumatic brain injury. Acta Neurologica Scandinavica, 1, 16-23.

14. Forslund, M. V., Roe, C., Sigurdardottir, S., \& Andelic, N. (2013). Predicting health-related quality of life 2 years after moderate-tosevere traumatic brain injury. Acta Neurologica Scandinavica, 4, 220-227.

15. Grauwmeijer, E., Heijenbrok-Kal, M. H., \& Ribbers, G. M. (2014). Health-related quality of life 3 years after moderate to severe traumatic brain injury: A prospective cohort study. Archives of Physical Medicine and Rehabilitation, 7, 1268-1276.
16. Williamson, M. L. C., Elliott, T. R., Berry, J. W., Underhill, A. T., Stavrinos, D., \& Fine, P. R. (2013). Predictors of health-related quality-of-life following traumatic brain injury. Brain Injury, 9, 992-999.

17. Maruish, M. E. (2011). User's manual for the SF-36v2 Health Survey, Quality Metric Incorporated.

18. Von Steinbuechel, N., Covic, A., Polinder, S., Kohlmann, T., Cepulyte, U., Poinstingl, H., Backhaus, J., Bakx, W., Bullinger, M., \& Christensen, A.-L. (2016). Assessment of health-related quality of life after TBI: Comparison of a disease-specific (QOLIBRI) with a generic (SF-36) instrument. Behavioural Neurology. https://doi.org/10.1155/2016/7928014

19. von Steinbüchel, N., Wilson, L., Gibbons, H., Hawthorne, G., Höfer, S., Schmidt, S., Bullinger, M., Maas, A., Neugebauer, E., \& Powell, J. (2010). Quality of Life after Brain Injury (QOLIBRI): Scale development and metric properties. Journal of Neurotrauma, 7, 1167-1185.

20. Truelle, J.-L., Koskinen, S., Hawthorne, G., Sarajuuri, J., Formisano, R., Von Wild, K., Neugebauer, E., Wilson, L., Gibbons, H., \& Powell, J. (2010). Quality of life after traumatic brain injury: The clinical use of the QOLIBRI, a novel disease-specific instrument. Brain Injury, 11, 1272-1291.

21. Maas, A. I. R., Menon, D. K., Adelson, P. D., Andelic, N., Bell, M. J., Belli, A., Bragge, P., Brazinova, A., Büki, A., \& Chesnut, R. M. (2017). Traumatic brain injury: Integrated approaches to improve prevention, clinical care, and research. Lancet Neurology, 16(12), 987-1048.

22. Ponsford, J., Willmott, C., Rothwell, A., Cameron, P., Kelly, A.-M., Nelms, R., Curran, C., \& Ng, K. I. M. (2000). Factors influencing outcome following mild traumatic brain injury in adults. Journal of the International Neuropsychological Society, $5,568-579$.

23. Lingsma, H. F., Yue, J. K., Maas, A. I. R., Steyerberg, E. W., Manley, G. T., \& TRACK-TBI Investigators (Cooper, S. R., DamsO'Connor, K., Gordon, W. A., \& Menon, D. K.). (2015). Outcome prediction after mild and complicated mild traumatic brain injury: External validation of existing models and identification of new predictors using the TRACK-TBI pilot study. Journal of Neurotrauma, 2, 83-94.

24. Steyerberg, E. W. (2008). Clinical prediction models: A practical approach to development, validation, and updating. Springer.

25. Dijkland, S. A., Foks, K. A., Polinder, S., Dippel, D. W. J., Maas, A., Lingsma, H., \& Steyerberg, E. W. (2019). Prognosis in moderate and severe traumatic brain injury: A systematic review of contemporary models and validation studies. Journal of Neurotrauma, 37(1), 1-13.

26. Corrigan, J. D., Bogner, J. A., Mysiw, W. J., Clinchot, D., \& Fugate, L. (2001). Life satisfaction after traumatic brain injury. Journal of Head Trauma Rehabilitation, 6, 543-555.

27. Klonoff, P. S., Costa, L. D., \& Snow, W. G. (1986). Predictors and indicators of quality of life in patients with closed-head injury. Journal of Clinical and Experimental Neuropsychology, $5,469-485$.

28. McCarthy, M. L., Dikmen, S. S., Langlois, J. A., Selassie, A. W., Gu, J. K., \& Horner, M. D. (2006). Self-reported psychosocial health among adults with traumatic brain injury. Archives of Physical Medicine and Rehabilitation, 7, 953-961.

29. Steadman-Pare, D., Colantonio, A., Ratcliff, G., Chase, S., \& Vernich, L. (2001). Factors associated with perceived quality of life many years after traumatic brain injury. Journal of Head Trauma Rehabilitation, 4, 330-342.

30. Warren, L., Wrigley, J. M., Yoels, W. C., \& Fine, P. R. (1996). Factors associated with life satisfaction among a sample of 
persons with neurotrauma. Journal of Rehabilitation Research and Development, 33(4), 404-408.

31. Webb, C. R., Wrigley, M., Yoels, W., \& Fine, P. R. (1995). Explaining quality of life for persons with traumatic brain injuries 2 years after injury. Archives of Physical Medicine and Rehabilitation, 12, 1113-1119.

32. Maas, A. I. R., Menon, D. K., Steyerberg, E. W., Citerio, G., Lecky, F., Manley, G. T., Hill, S., Legrand, V., \& Sorgner, A. (2014). Collaborative European NeuroTrauma Effectiveness Research in Traumatic Brain Injury (CENTER-TBI) a prospective longitudinal observational study. Neurosurgery, 1, 67-80.

33. Steyerberg, E. W., Wiegers, E., Sewalt, C., Buki, A., Citerio, G., De Keyser, V., Ercole, A., Kunzmann, K., Lanyon, L., \& Lecky, F. (2019). Case-mix, care pathways, and outcomes in patients with traumatic brain injury in CENTER-TBI: A European prospective, multicentre, longitudinal, cohort study. Lancet Neurology, 10, 923-934.

34. Vickery, C. D., Gontkovsky, S. T., \& Caroselli, J. S. (2005). Selfconcept and quality of life following acquired brain injury: A pilot investigation. Brain Injury, 9, 657-665.

35. Teasdale, G., Maas, A., Lecky, F., Manley, G., Stocchetti, N., \& Murray, G. (2014). The Glasgow Coma Scale at 40 years: Standing the test of time. Lancet Neurology, 8, 844-854.

36. Van Leeuwen, N., Lingsma, H., Perel, P., Lecky, F., Roozenbeek, R., Lu, J., Shakur, H., Weir, J., Steyerberg, E. W., \& Maas A. I. R. (2011). Prognostic value of major extracranial injury in traumatic brain injury: An individual patient data meta-analysis in 39,274 patients. Neurosurgery, 4, 811-818.

37. Van Buuren, S., \& Groothuis-Oudshoorn, K. (2010). mice: Multivariate imputation by chained equations in R. Journal of Statistical Software, 45(3), 1-68.

38. Von Steinbüchel, N., Wilson, L., Gibbons, H., Hawthorne, G., Höfer, S., Schmidt, S., Bullinger, M., Maas, A., Neugebauer, E., \& Powell, J. (2010). Quality of Life after Brain Injury (QOLIBRI): Scale validity and correlates of quality of life. Journal of Neurotrauma, 7, 1157-1165.

39. Wilson, L., Marsden-Loftus, I., Koskinen, S., Bakx, W., Bullinger, M., Formisano, R., Maas, A., Neugebauer, E., Powell, J., \& Sarajuuri, J. (2017). Interpreting quality of life after brain injury scores: Cross-walk with the short form-36. Journal of Neurotrauma, 1, 59-65.

40. R Core Team. (2013). R: A language and environment for statistical computing. R Foundation for Statistical Computing.

41. Harrell, F. E. Jr. (2012). rms: Regression Modeling Strategies. R package version 3.4-0.

42. Moons, K. G. M., Altman, D. G., Reitsma, J. B., \& Collins, G. S. (2015). New guideline for the reporting of studies developing, validating, or updating a multivariable clinical prediction model: The TRIPOD statement. Annals of Internal Medicine, 5, 303-305.

43. Morton, M. V., \& Wehman, P. (1995). Psychosocial and emotional sequelae of individuals with traumatic brain injury: A literature review and recommendations. Brain Injury, 1, 81-92.

44. Williams, D. H., Levin, H. S., \& Eisenberg, H. M. (1990). Mild head injury classification. Neurosurgery, 3, 422-428.

45. Voormolen, D. C., Haagsma, J. A., Polinder, S., Maas, A. I. R., Steyerberg, E. W., Vuleković, P., Sewalt, C. A., Gravesteijn, B. Y., Covic, A., \& Andelic, N. (2019). Post-concussion symptoms in complicated vs. uncomplicated mild traumatic brain injury patients at three and six months post-injury: Results from the CENTER-TBI study. Journal of Clinical Medicine, 11, 1921.

46. Summaka, M., Zein, H., Elias, E., Naim, I., Fares, Y., \& Nasser, Z. (2020). Prediction of quality of life by Helsinki computed tomography scoring system in patients with traumatic brain injury. Brain Injury, 9, 1229-1236.

47. Curran, C. A., Ponsford, J. L., \& Crowe, S. (2000). Coping strategies and emotional outcome following traumatic brain injury: A comparison with orthopedic patients. Journal of Head Trauma Rehabilitation, 6, 1256-1274.

48. Anson, K., \& Ponsford, J. (2006). Coping and emotional adjustment following traumatic brain injury. Journal of Head Trauma Rehabilitation, 3, 248-259.

49. Rainey, E. E., Petrey, L. B., Reynolds, M., Agtarap, S., \& Warren, A. M. (2014). Psychological factors predicting outcome after traumatic injury: The role of resilience. American Journal of Surgery, $4,517-523$

50. Tomberg, T., Toomela, A., Pulver, A., \& Tikk, A. (2005). Coping strategies, social support, life orientation and health-related quality of life following traumatic brain injury. Brain Injury, 14, 1181-1190.

51. Voormolen, D. C., Polinder, S., Von Steinbuechel, N., Vos, P. E., Cnossen, M. C., \& Haagsma, J. A. (2019). The association between post-concussion symptoms and health-related quality of life in patients with mild traumatic brain injury. Injury, 5 , 1068-1074.

52. Wilson, L., Horton, L., Kunzmann, K., Sahakian, B. J., Newcombe, V. F. J., Stamatakis, E. A., von Steinbuechel, N., Cunitz, K., Covic, A., \& Maas, A. (2020). Understanding the relationship between cognitive performance and function in daily life after traumatic brain injury. Journal of Neurology, Neurosurgery and Psychiatry. https://doi.org/10.1136/jnnp-2020-324492

Publisher's Note Springer Nature remains neutral with regard to jurisdictional claims in published maps and institutional affiliations.

\section{Authors and Affiliations}

\section{Isabel R. A. Retel Helmrich ${ }^{1,8}$ - David van Klaveren ${ }^{1,2,8} \cdot$ Simone A. Dijkland $^{1,8} \cdot$ Hester F. Lingsma ${ }^{1,8}$. Suzanne Polinder ${ }^{1,8} \cdot$ Lindsay Wilson $^{5} \cdot$ Nicole von Steinbuechel $^{3}$. Joukje van der Naalt ${ }^{4}$. Andrew I. R. Maas ${ }^{6}$. Ewout W. Steyerberg ${ }^{1,7}$. CENTER-TBI Collaborators}

David van Klaveren

d.vanklaveren@erasmusmc.nl

Simone A. Dijkland

s.dijkland@erasmusmc.nl

Hester F. Lingsma

h.lingsma@erasmusmc.nl

Suzanne Polinder

s.polinder@erasmusmc.nl
Lindsay Wilson

1.wilson@stir.ac.uk

Nicole von Steinbuechel

nvsteinbuechel@med.uni-goettingen.de

Joukje van der Naalt

j.van.der.naalt@umcg.nl

Andrew I. R. Maas

Andrew.Maas@uza.be 
Ewout W. Steyerberg

e.steyerberg@erasmusmc.nl

1 Department of Public Health, Center for Medical Decision Making, Erasmus MC-University Medical Center Rotterdam, Rotterdam, The Netherlands

2 Predictive Analytics and Comparative Effectiveness Center, Institute for Clinical Research and Health Policy Studies/Tufts Medical Center, Boston, USA

3 Department of Medical Psychology and Medical Sociology, Georg-August-University, 37073 Göttingen, Germany
4 University Medical Center Groningen, University of Groningen, 9713 GZ Groningen, The Netherlands

5 Division of Psychology, University of Stirling, Stirling FK9 4LA, UK

6 Department of Neurosurgery, University Hospital Antwerp and University of Antwerp, Edegem, 2650 Antwerp, Belgium

7 Department of Biomedical Data Sciences, Leiden University Medical Center, PO Box 9600, 2300 RC Leiden, The Netherlands

8 Department of Public Health, Erasmus MC University Medical Center, PO Box 2040, 3000 CA Rotterdam, The Netherlands 